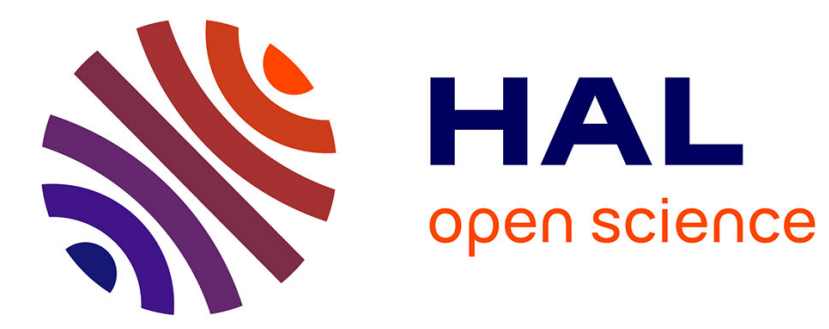

\title{
Asymptotic analysis for domains separated by a thin layer made of periodic vertical beams
}

Georges Griso, Anastasia Migunova, Julia Orlik

\section{To cite this version:}

Georges Griso, Anastasia Migunova, Julia Orlik. Asymptotic analysis for domains separated by a thin layer made of periodic vertical beams. 2016. hal-01449381

\section{HAL Id: hal-01449381 \\ https://hal.science/hal-01449381}

Preprint submitted on 30 Jan 2017

HAL is a multi-disciplinary open access archive for the deposit and dissemination of scientific research documents, whether they are published or not. The documents may come from teaching and research institutions in France or abroad, or from public or private research centers.
L'archive ouverte pluridisciplinaire HAL, est destinée au dépôt et à la diffusion de documents scientifiques de niveau recherche, publiés ou non, émanant des établissements d'enseignement et de recherche français ou étrangers, des laboratoires publics ou privés. 


\title{
Asymptotic analysis for domains separated by a thin layer made of periodic vertical beams
}

\author{
Georges Griso, Anastasia Migunova, Julia Orlik
}

\begin{abstract}
We consider a thin heterogeneous layer consisting of thin beams (of radius $r$ ) and study the limit behaviour of this problem as the period $\varepsilon$, the thickness $\delta$ and the radius $r$ of the beams tend to zero. The decomposition of the displacement field into beams developed in [10] is used, which allows to obtain a priori estimates. Two types of unfolding operators are introduced to deal with different parts of the decomposition. In conclusion, we obtain the limit problem together with transmission conditions across the interface.
\end{abstract}

KEY WORDS: linear elasticity, junctions, decomposition of beam displacements, interface, homogenization.

Mathematics Subject Classification (2010): 74B05, 74K10, 74K30, 74Q05, 74A50, 35B27.

\section{Introduction}

In this paper a system of elasticity equations in domains separated by a thin heterogeneous layer is considered. The layer is composed of periodically distributed vertical beams, whose diameter and height tend to zero together with the period of the structure. The structure is fixed on the bottom. We consider the case of the isotropic linearized elasticity system.

Elasticity problems involving thin layers with periodic heterogeneous structure appear in many technical applications, where special constraints on the stiffness or compliance of a textile are required, depending on the type of application. For example, drainages, spacer fabrics in car-seats and protective wear, working for the outer-plane compression, should provide a certain stiffness and strength against an external mechanical loading. Thin layers were considered in a number of papers (see e.g. [6, 7, 9, 12]). In particular, [7] deals with a layer composed of a material with holes, whose size is scaled with an additional small parameter; [9, 12] consider the case of the layer whose elastic coefficients are of the same order as its thickness. The thin beams and their junction with 3D structures were studied in $[1,2,3,10,11]$ : [10] deals with the decomposition for a single beam; in [11] the structure made of curved beams is considered. [2,3] study the limit behavior of structures composed of rods in a junction with a plate.

We consider 3 small parameters: the thickness $\delta$ of the layer (and the height of the beams at the same time), the radius $r$ of the rods and the period of the layer $\varepsilon$ (see Fig. 1(a)). The first problem with this structure arises when we obtain estimates on displacements. To overcome this difficulty we used a decomposition of the thin beams in the mean displacement and the rotation of the cross-section which was introduced in [10]. After deriving estimates for the components of the decomposition we obtain bounds for the minimizing sequence which depend on $\varepsilon, r, \delta$. The result leads to studying three critical cases with different ratios between small parameters. Two of them are considered in the present paper and lead to the same kind of limit problem. The third one corresponds no longer to thin beams but to small inclusions and is therefore not studied in the present paper.

The main result of the work is the limit problem (5.7), which replaces the structural layer by a kind of the linearized contact conditions. There $\widetilde{F}_{\alpha}^{m}$ is the volume force, applied to the structural layer, $E^{m}$ are the Young's moduli of the beams, $\left[u_{\alpha}^{ \pm}\right]_{\mid \Sigma}$ are the macroscopic interface jumps in the displacement, and the convolution kernel $K_{\alpha}$ is the fundamental solution of the beam problem (5.9).

In order to obtain the limit problem, the periodic unfolding method, applied to the components of the decomposition, is used. Basic theory on the unfolding method can be found in [8]. The idea of the method is to replace integrals of functions over domains depending on small parameters (e.g., oscillating, like in the periodic case, or thin domains) by the integrals over several finite domains. This is done by a successive substitution, leading to the increasing of the problem's dimension, and a change of variables. In the present study we introduce two additional types of the unfolding operators in order to deal with the mean displacement and rotation which depend only on component $x_{3}$ and the warping which depends on all $\left(x_{1}, x_{2}, x_{3}\right)$. In the limit we derive a $3 \mathrm{D}$ elasticity problem for two domains separated by an interface with the inhomogeneous Robin-type condition on it. The coefficients in this jump condition are obtained from the solution of auxiliary 1D beam problems.

The paper is organized as follows. In Section 2 the geometry and the weak and strong formulations of the problem are introduced. Section 3 presents the decomposition of a single beam and the a priori estimates. In Section 4, the periodic unfolding operators are introduced and their properties are defined. Section 5 deals with taking the limit and obtaining the variational formulation for the limit problem. Section 6 contains an additional information and a numerical illustration. 


\section{Problem statement}

\subsection{Geometry}

In the Euclidean space $\mathbb{R}^{2}$ let $\omega$ be a connected domain with a Lipschitz boundary and let $L>0$ be a fixed real number. Define the reference domains:

$$
\begin{array}{ll}
\Omega^{-} & =\omega \times(-L, 0), \\
\Omega^{+} & =\omega \times(0, L), \\
\Sigma & =\omega \times\{0\} .
\end{array}
$$

Moreover, $\Omega$ (see Figure 1b) is defined by

$$
\Omega=\Omega^{+} \cup \Omega^{-} \cup \Sigma=\omega \times(-L, L) .
$$

For the domains corresponding to structure with a layer of thickness $\delta$ we introduce the following notations:

$$
\begin{aligned}
\Omega_{\delta}^{+} & =\omega \times(\delta, L), \\
\Sigma_{\delta}^{+} & =\omega \times\{\delta\} .
\end{aligned}
$$

In order to describe the configuration of the layer, for any $(d, r) \in(0,+\infty)^{2}$ we define the rod $B_{r, d}$ by

$$
B_{r, d}=D_{r} \times(0, d),
$$

where $D_{r}=D(O, r)$ is the disc of center $O$ and radius $r$.

The set of rods is

$$
\Omega_{r, \varepsilon, \delta}^{i}=\bigcup_{\mathbf{i} \in \widehat{\Xi}_{\varepsilon} \times\{0\}}\left\{x \in \mathbb{R}^{3} \mid x \in \mathbf{i} \varepsilon+B_{r, \delta}\right\}
$$

where

$$
\widehat{\Xi}_{\varepsilon}=\left\{\xi \in \mathbb{Z}^{2} \mid \varepsilon(\xi+Y) \subset \omega\right\}, \quad Y=\left(-\frac{1}{2} ; \frac{1}{2}\right)^{2} .
$$

Almost any $z \in \mathbb{R}^{2}$ can be decomposed in the way $z=[z]_{Y}+\{z\}_{Y}$, where $[z]_{Y} \in \mathbb{Z}^{2}$, and $\{z\}_{Y} \in Y$.

Moreover, we set:

$$
\begin{gathered}
\widehat{\omega}_{\varepsilon}=\text { interior } \\
\bigcup_{\substack{\mathbf{i} \in \widehat{\Xi}_{\varepsilon} \\
\Lambda_{\varepsilon}=\omega \backslash \widehat{\omega}_{\varepsilon} .}} \varepsilon(\mathbf{i}+\bar{Y}),
\end{gathered}
$$

The physical reference configuration (see Figure 1a) is defined by $\Omega_{r, \varepsilon, \delta}$ :

$$
\Omega_{r, \varepsilon, \delta}=\operatorname{interior}\left(\overline{\Omega^{-}} \cup \overline{\Omega_{r, \varepsilon, \delta}^{i}} \cup \overline{\Omega_{\delta}^{+}}\right) .
$$

The structure is fixed on a part $\Gamma$ with the non-zero measure of the boundary $\partial \Omega^{-} \backslash \Sigma$.

We make the following assumptions:

$$
r<\frac{\varepsilon}{2}, \quad \frac{r}{\delta} \leq C .
$$

Here, the first assumption (2.7) is a beam separation in the reference configuration for the beams whereas with the second one, we want to eliminate the case $\frac{\delta}{r} \rightarrow 0$ which requires the use of tools for plates (see [10]).

\subsection{Strong formulation}

Choose an isotropic material with Lamé constants $\lambda^{m}, \mu^{m}$ for the beams and another isotropic material with Lamé constants $\lambda^{b}, \mu^{b}$ for $\Omega^{-}$and $\Omega_{\delta}^{+}$. Then we have the following values for the Poisson's coefficient of the material and Young's modulus:

$$
\begin{gathered}
\nu^{m}=\frac{\lambda^{m}}{2\left(\lambda^{m}+\mu^{m}\right)}, \quad \nu^{b}=\frac{\lambda^{b}}{2\left(\lambda^{b}+\mu^{b}\right)}, \\
E^{m}=\frac{\mu^{m}\left(3 \lambda^{m}+2 \mu^{m}\right)}{\lambda^{m}+\mu^{m}}, \quad E^{b}=\frac{\mu^{b}\left(3 \lambda^{b}+2 \mu^{b}\right)}{\lambda^{b}+\mu^{b}} .
\end{gathered}
$$




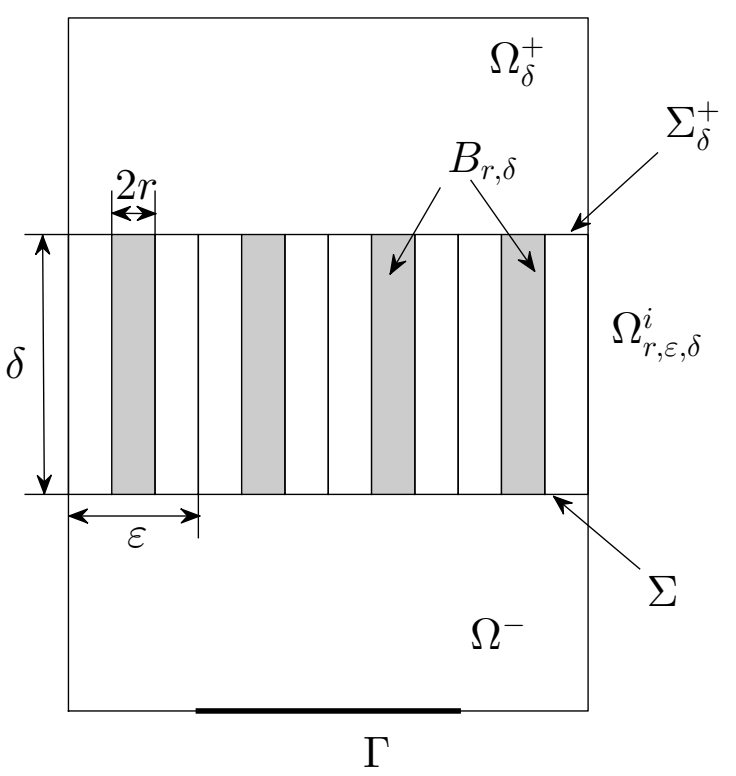

(a) The domain with the thin layer

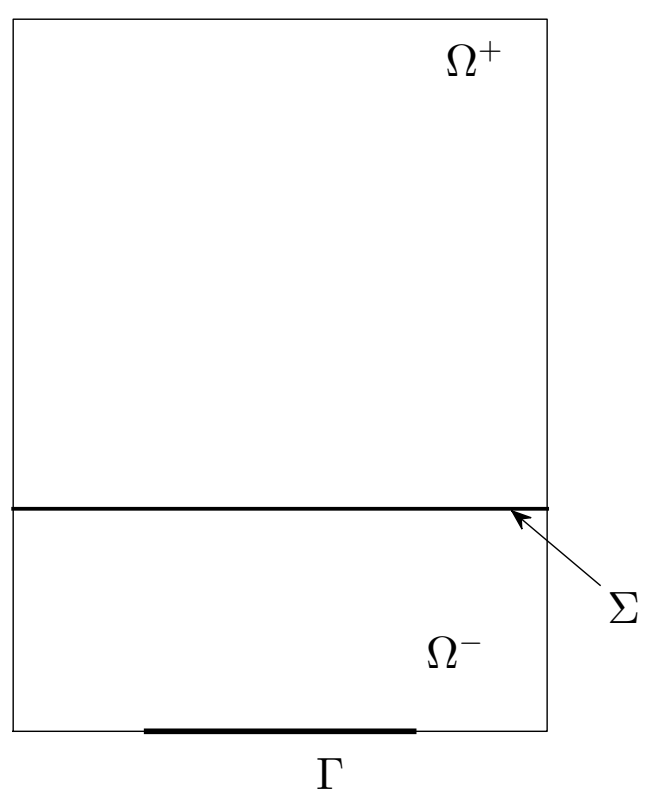

(b) The limit problem

Figure 1: The reference configuration

The linearized strain tensor or symmetric deformation field is defined by

$$
e(u)=\frac{\nabla u+\nabla^{T} u}{2}
$$

The Cauchy stress tensor in $\Omega_{r, \varepsilon, \delta}$ is linked to $e\left(u_{r, \varepsilon, \delta}\right)$ through the standard Hooke's law:

$$
\sigma_{r, \varepsilon, \delta}= \begin{cases}\lambda^{b}\left(\operatorname{Tr} e\left(u_{r, \varepsilon, \delta}\right)\right) I+2 \mu^{b} e\left(u_{r, \varepsilon, \delta}\right) & \text { in } \Omega^{-} \cup \Omega_{\delta}^{+}, \\ \lambda^{m}\left(\operatorname{Tr} e\left(u_{r, \varepsilon, \delta}\right)\right) I+2 \mu^{m} e\left(u_{r, \varepsilon, \delta}\right) & \text { in } \Omega_{r, \varepsilon, \delta}^{i} .\end{cases}
$$

We consider the standard linear equations of elasticity in $\Omega_{r, \varepsilon, \delta}$. The unknown displacement $u_{r, \varepsilon, \delta}: \Omega_{r, \varepsilon, \delta} \rightarrow$ $\mathbb{R}^{3}$ satisfies the following problem:

$$
\begin{cases}\nabla \cdot \sigma_{r, \varepsilon, \delta}=-f_{r, \varepsilon, \delta} & \text { in } \Omega_{r, \varepsilon, \delta} \\ u_{r, \varepsilon, \delta}=0 & \text { on } \Gamma \\ \sigma_{r, \varepsilon, \delta} \cdot \nu=0 & \text { on } \partial \Omega_{r, \varepsilon, \delta} \backslash \Gamma\end{cases}
$$

\subsection{Weak formulation}

If $\mathbb{V}$ denotes the space

$$
\mathbb{V}=\left\{v \in H^{1}\left(\Omega_{r, \varepsilon, \delta} ; \mathbb{R}^{3}\right) \mid v=0 \text { on } \Gamma\right\},
$$

the variational formulation of $(2.8)$ is

$$
\left\{\begin{array}{l}
\text { Find } u_{r, \varepsilon, \delta} \in \mathbb{V}, \\
\int_{\Omega_{r, \varepsilon, \delta}} \sigma_{r, \varepsilon, \delta}: e(\varphi) d x=\int_{\Omega_{r, \varepsilon, \delta}} f_{r, \varepsilon, \delta} \cdot \varphi d x, \quad \forall \varphi \in \mathbb{V} .
\end{array}\right.
$$

Throughout the paper and for any $v \in \mathbb{V}$ we denote by

$$
\sigma(v)=\lambda(\operatorname{Tr} e(v)) I+2 \mu e(v)= \begin{cases}\lambda^{m}(\operatorname{Tr} e(v)) I+2 \mu^{m} e(v) & \text { in } \Omega^{-} \cup \Omega_{\delta}^{+}, \\ \lambda^{b}(\operatorname{Tr} e(v)) I+2 \mu^{b} e(v) & \text { in } \Omega_{r, \varepsilon, \delta}^{i}\end{cases}
$$

and

$$
\mathcal{E}(v)=\int_{\Omega_{r, \varepsilon, \delta}} \sigma(v): e(v) d x
$$

the total elastic energy of the displacement $v$. Indeed choosing $v=u_{r, \varepsilon, \delta}$ in (2.9) leads to the usual energy relation

$$
\mathcal{E}\left(u_{r, \varepsilon, \delta}\right)=\int_{\Omega_{r, \varepsilon, \delta}} f_{r, \varepsilon, \delta} \cdot u_{r, \varepsilon, \delta} d x
$$


We equip the space $\mathbb{V}$ with the following norm:

$$
\|u\|_{V}=\|e(u)\|_{L^{2}\left(\Omega_{r, \varepsilon, \delta}\right)} .
$$

In the next step we derive the Korn estimates for each sub-domain. Since $\Omega^{-}$touches the Dirichlet boundary, the 3D-Korn inequality for this sub-domain is the following:

$$
\|u\|_{H^{1}\left(\Omega^{-}\right)} \leq C\|e(u)\|_{L^{2}\left(\Omega^{-}\right)} .
$$

\section{Decomposition of displacements in the periodic layer with beams}

\subsection{Displacement of a single beam. Preliminary estimates}

To obtain a priori estimates on $u_{r, \varepsilon, \delta}$ and $e\left(u_{r, \varepsilon, \delta}\right)_{S}$ we will need Korn's inequalities for this type of domain. However, for a multi-structured domains, it is not convenient to estimate the constant in a Korn's type inequality, because components of the displacement field may be of different orders of magnitude. To overcome this difficulty, we will use a decomposition for the displacements of the beams. A displacement of the beam $B_{r, d}$ is decomposed as the sum of three fields: the first one stands for the displacement of the center line, the second one stands for the rotations of the cross sections and the last one is the warping, which takes into account the deformations of the cross sections.

We recall the definition of the elementary displacement from [10].

Definition 3.1. The elementary displacement $U_{e}$, associated with $u \in L^{1}\left(B_{r, d}, \mathbb{R}^{3}\right)$, is given by

$$
U_{e}\left(x_{1}, x_{2}, x_{3}\right)=\mathcal{U}\left(x_{3}\right)+\mathcal{R}\left(x_{3}\right) \wedge\left(x_{1} \mathbf{e}_{1}+x_{2} \mathbf{e}_{2}\right), \quad \text { for a.e. } x=\left(x_{1}, x_{2}, x_{3}\right) \in B_{r, d},
$$

where

$$
\left\{\begin{array}{l}
\mathcal{U}=\frac{1}{\pi r^{2}} \int_{D_{r}} u\left(x_{1}, x_{2}, \cdot\right) d x_{1} d x_{2}, \\
\mathcal{R}_{3}=\frac{1}{\left(I_{1}+I_{2}\right) r^{4}} \int_{D_{r}}\left(x_{1} u_{2}\left(x_{1}, x_{2}, \cdot\right)-x_{2} u_{1}\left(x_{1}, x_{2}, \cdot\right)\right) d x_{1} d x_{2}, \\
\mathcal{R}_{\alpha}=\frac{(-1)^{3-\alpha}}{I_{3-\alpha} r^{4}} \int_{D_{r}} x_{3-\alpha} u_{3}\left(x_{1}, x_{2}, \cdot\right) d x_{1} d x_{2}, \quad I_{\alpha}=\int_{D_{1}} x_{\alpha}^{2} d x_{1} d x_{2}=\frac{\pi}{4} .
\end{array}\right.
$$

We write

$$
\bar{u}=u-U_{e} .
$$

The displacement $\bar{u}$ is the warping. Note that

$$
\begin{aligned}
& \int_{D_{r}} \bar{u}\left(x_{1}, x_{2}, \cdot\right) d x_{1} d x_{2}=0, \\
& \int_{D_{r}}\left(x_{1} \bar{u}_{2}\left(x_{1}, x_{2}, \cdot\right)-x_{2} \bar{u}_{1}\left(x_{1}, x_{2}, \cdot\right)\right) d x_{1} d x_{2}=0, \\
& \int_{D_{r}} x_{1} \bar{u}_{3}\left(x_{1}, x_{2}, \cdot\right) d x_{1} d x_{2}=\int_{D_{r}} x_{2} \bar{u}_{3}\left(x_{1}, x_{2}, \cdot\right) d x_{1} d x_{2}=0 .
\end{aligned}
$$

The following theorem is proved in [10].

Theorem 3.1. Let $u$ be in $H^{1}\left(B_{r, d} ; \mathbb{R}^{3}\right)$ and let $u=U_{e}+\bar{u}$ be the decomposition of $u$ given by (3.1)-(3.3). There exists a constant $C$ independent of $r$ such that the following estimates hold:

$$
\begin{aligned}
& \|\bar{u}\|_{L^{2}\left(B_{r, d}\right)} \leq C r\|e(u)\|_{L^{2}\left(B_{r, d}\right)}, \quad\|\nabla \bar{u}\|_{L^{2}\left(B_{r, d}\right)} \leq C\|e(u)\|_{L^{2}\left(B_{r, d}\right)}, \\
& \left\|\frac{d \mathcal{R}}{d x_{3}}\right\|_{L^{2}(0, d)} \leq \frac{C}{r^{2}}\|e(u)\|_{L^{2}\left(B_{r, d}\right)}, \\
& \left\|\frac{d \mathcal{U}}{d x_{3}}-\mathcal{R} \wedge \mathbf{e}_{3}\right\|_{L^{2}(0, d)} \leq \frac{C}{r}\|e(u)\|_{L^{2}\left(B_{r, d}\right)} .
\end{aligned}
$$

We set

$$
Y_{\varepsilon}=\varepsilon Y, \quad W_{\varepsilon}=Y_{\varepsilon} \times(-\varepsilon, 0), \quad B_{r, \varepsilon}^{\prime}=D_{r} \times(-\varepsilon, 0), \quad W_{r, \varepsilon, \delta}^{\prime}=W_{\varepsilon} \cup D_{r} \times(-\varepsilon, \delta) .
$$


Lemma 3.1. Let $u$ be in $H^{1}\left(W_{r, \varepsilon, \delta}^{\prime}, \mathbb{R}^{3}\right)$ and let $u=U_{e}+\bar{u}$ be the decomposition of the restriction of $u$ to the rod $B_{r, \varepsilon}^{\prime}$ given by (3.1)-(3.3). There exists a constant $C$ independent of $\delta, \varepsilon$ and $r$ such that the following estimates hold:

$$
\begin{aligned}
& |\mathcal{R}(0)|^{2} \leq \frac{C}{r^{3}}\|\nabla u\|_{L^{2}\left(W_{\varepsilon}\right)}^{2}, \\
& \|\mathcal{R}\|_{L^{2}(0, \delta)}^{2} \leq C \frac{\delta}{r^{3}}\|\nabla u\|_{L^{2}\left(W_{\varepsilon}\right)}^{2}+C \frac{\delta^{2}}{r^{4}}\|e(u)\|_{L^{2}\left(B_{r, \delta}\right)}^{2}, \\
& \left\|\frac{d \mathcal{U}_{\alpha}}{d x_{3}}\right\|_{L^{2}(0, \delta)}^{2} \leq C \frac{\delta}{r^{3}}\|\nabla u\|_{L^{2}\left(W_{\varepsilon}\right)}^{2}+C \frac{\delta^{2}}{r^{4}}\|e(u)\|_{L^{2}\left(B_{r, \delta}\right)}^{2}, \\
& \left\|\mathcal{U}_{3}-\mathcal{U}_{3}(0)\right\|_{L^{2}(0, \delta)}^{2} \leq C \frac{\delta^{2}}{r^{2}}\|e(u)\|_{L^{2}\left(B_{r, \delta}\right)}^{2}, \\
& \left\|\mathcal{U}_{\alpha}-\mathcal{U}_{\alpha}(0)\right\|_{L^{2}(0, \delta)}^{2} \leq C \frac{\delta^{3}}{r^{3}}\|\nabla u\|_{L^{2}\left(W_{\varepsilon}\right)}^{2}+C \frac{\delta^{4}}{r^{4}}\|e(u)\|_{L^{2}\left(B_{r, \delta}\right)}^{2}, \\
& \|u(\cdot, \cdot, 0)-\mathcal{U}(0)\|_{L^{2}\left(Y_{\varepsilon}\right)}^{2} \leq C \varepsilon\|\nabla u\|_{L^{2}\left(W_{\varepsilon}\right)}^{2}+C \frac{\varepsilon^{2}}{r}\|e(u)\|_{L^{2}\left(W_{\varepsilon}\right)}^{2} .
\end{aligned}
$$

A proof of the lemma is given in Appendix A.

\subsection{A priori Korn-type estimates}

In this section all the constants do not depend on $\varepsilon, \delta$ and $r$. We denote by $x^{\prime}=\left(x_{1}, x_{2}\right)$ a point in $\mathbb{R}^{2}$.

\subsubsection{Decomposition of displacements in the layer with beams on the in-plane and outer-plane components}

We decompose the displacement $u \in \mathbb{V}$ in each beam $\varepsilon \mathbf{i}+B_{r, \delta}, \mathbf{i} \in \widehat{\Xi}_{\varepsilon} \times\{0\}$ as in the Definition 3.1. The components of the elementary displacement are denoted $\mathcal{U}_{\xi}, \mathcal{R}_{\xi}$, where $\xi=\left[\frac{x^{\prime}}{\varepsilon}\right]_{Y}$.

Now we define the fields $\widetilde{\mathcal{U}}, \widetilde{\mathcal{R}}$ and $\widetilde{u}$ for a.e. $x \in B_{r, \delta}, s \in \omega$ by

$$
\begin{aligned}
& \widetilde{\mathcal{U}}\left(s_{1}, s_{2}, x_{3}\right)=\left\{\begin{array}{lr}
\mathcal{U}_{\xi}\left(x_{3}\right), & \text { if } \xi=\left[\frac{s}{\varepsilon}\right]_{Y} \in \widehat{\Xi}_{\varepsilon} \\
0, & \text { if } \xi \notin \widehat{\Xi}_{\varepsilon}
\end{array}, \quad \widetilde{\mathcal{R}}\left(s_{1}, s_{2}, x_{3}\right)=\left\{\begin{array}{ll}
\mathcal{R}_{\xi}\left(x_{3}\right), & \text { if } \xi=\left[\frac{s}{\varepsilon}\right]_{Y} \in \widehat{\Xi}_{\varepsilon} \\
0, & \text { if } \xi \notin \widehat{\Xi}_{\varepsilon}
\end{array},\right.\right. \\
& \widetilde{u}\left(s_{1}, s_{2}, x\right)=\left\{\begin{array}{lr}
\bar{u}_{\xi}(x), & \text { if } \xi=\left[\frac{s}{\varepsilon}\right]_{Y} \in \widehat{\Xi}_{\varepsilon} \\
0, & \text { if } \xi \notin \widehat{\Xi}_{\varepsilon}
\end{array} .\right.
\end{aligned}
$$

We have

$$
\widetilde{\mathcal{U}}, \widetilde{\mathcal{R}} \in L^{2}\left(\omega, H^{1}\left((0, \delta), \mathbb{R}^{3}\right)\right), \quad \widetilde{u} \in L^{2}\left(\omega, H^{1}\left(B_{r, \delta}, \mathbb{R}^{3}\right)\right)
$$

Moreover,

$$
\begin{aligned}
\|\widetilde{\mathcal{U}}\|_{L^{2}(\omega \times(0, \delta))}^{2}= & \varepsilon^{2} \sum_{\xi \in \hat{\Xi}_{\varepsilon}}\left\|\mathcal{U}_{\xi}\right\|_{L^{2}(0, \delta)}^{2}, \quad\|\widetilde{\mathcal{R}}\|_{L^{2}(\omega \times(0, \delta))}^{2}=\varepsilon^{2} \sum_{\xi \in \hat{\Xi}_{\varepsilon}}\left\|\mathcal{R}_{\xi}\right\|_{L^{2}(0, \delta)}^{2}, \\
& \|\widetilde{u}\|_{L^{2}\left(\omega \times B_{r, \delta}\right)}^{2}=\varepsilon^{2} \sum_{\xi \in \widehat{\Xi}_{\varepsilon}}\left\|\bar{u}_{\xi}\right\|_{L^{2}\left(B_{r, \delta}\right)}^{2} .
\end{aligned}
$$

As a consequence of Theorem 3.1 and Lemma 3.1 we get

Lemma 3.2. Let $u$ be in $\mathbb{V}$. The following estimates hold:

$$
\begin{aligned}
& \left\|\frac{\partial \widetilde{\mathcal{R}}}{\partial x_{3}}\right\|_{L^{2}(\omega \times(0, \delta))} \leq C \frac{\varepsilon}{r^{2}}\|u\|_{V}, \\
& \left\|\frac{\partial \widetilde{\mathcal{U}}}{\partial x_{3}}-\widetilde{\mathcal{R}} \wedge e_{3}\right\|_{L^{2}(\omega \times(0, \delta))} \leq C \frac{\varepsilon}{r}\|u\|_{V}, \\
& \left\|\nabla_{x} \widetilde{u}\right\|_{L^{2}\left(\omega \times B_{r, \delta}\right)} \leq C \varepsilon\|u\|_{V}, \\
& \|\widetilde{u}\|_{L^{2}\left(\omega \times B_{r, \delta}\right)} \leq C \varepsilon r\|u\|_{V}, \\
& \|\widetilde{\mathcal{R}}\|_{L^{2}(\omega \times(0, \delta))} \leq C \frac{\varepsilon \delta}{r^{2}}\|u\|_{V}, \\
& \left\|\frac{\partial \widetilde{\mathcal{U}}_{\alpha}}{\partial x_{3}}\right\|_{L^{2}(\omega \times(0, \delta))} \leq C \frac{\varepsilon \delta}{r^{2}}\|u\|_{V} .
\end{aligned}
$$


Moreover,

$$
\begin{aligned}
& \|\widetilde{\mathcal{R}}(\cdot, \cdot, 0)\|_{L^{2}\left(\widehat{\omega}_{\varepsilon}\right)}^{2} \leq C \frac{\varepsilon^{2}}{r^{3}}\|u\|_{V}^{2}, \\
& \|\widetilde{\mathcal{R}}(\cdot, \cdot, \delta)\|_{L^{2}\left(\widehat{\omega}_{\varepsilon}\right)}^{2} \leq C \frac{\varepsilon^{2}}{r^{3}}\|\nabla u\|_{L^{2}\left(\Omega_{\delta}^{+}\right)}^{2}, \\
& \left\|\widetilde{\mathcal{U}}_{3}-\widetilde{\mathcal{U}}_{3}(\cdot, \cdot, 0)\right\|_{L^{2}(\omega \times(0, \delta))} \leq C \frac{\delta \varepsilon}{r}\|u\|_{V}, \\
& \left\|\widetilde{\mathcal{U}}_{\alpha}-\widetilde{\mathcal{U}}_{\alpha}(\cdot, \cdot, 0)\right\|_{L^{2}(\omega \times(0, \delta))} \leq C \frac{\delta^{2} \varepsilon}{r^{2}}\|u\|_{V}, \quad \text { where } \alpha=1,2 .
\end{aligned}
$$

The proof of estimates $(3.7)_{1}-(3.7)_{6}$ follows directly from $(2.11),(3.5)_{3},(3.5)_{4}$ and $(3.6)_{2}-(3.6)_{3}$, and the estimates (3.8) are the consequences of the estimates in Lemma 3.1 and (2.11).

\subsubsection{Estimates on interface traces}

Lemma 3.3. There exists a constant $C$ independent of $\varepsilon, \delta, r$ such that for any $u \in \mathbb{V}$

$$
\begin{gathered}
\|u(\cdot, \cdot, 0)-\widetilde{\mathcal{U}}(\cdot, \cdot, 0)\|_{L^{2}\left(\widehat{\omega}_{\varepsilon}\right)}^{2} \leq C \frac{\varepsilon^{2}}{r}\|u\|_{V}^{2}, \\
\|u(\cdot, \cdot, \delta)-\tilde{\mathcal{U}}(\cdot, \cdot, \delta)\|_{L^{2}\left(\widehat{\omega}_{\varepsilon}\right)}^{2} \leq C \varepsilon\|\nabla u\|_{L^{2}\left(\Omega_{\delta}^{+}\right)}^{2}+C \frac{\varepsilon^{2}}{r}\|u\|_{V}^{2} .
\end{gathered}
$$

Moreover,

$$
\begin{gathered}
\left\|u_{\alpha}(\cdot, \cdot, \delta)-u_{\alpha}(\cdot, \cdot, 0)\right\|_{L^{2}\left(\widehat{\omega}_{\varepsilon}\right)}^{2} \leq C \varepsilon\|\nabla u\|_{L^{2}\left(\Omega_{\delta}^{+}\right)}^{2}+C \frac{\varepsilon^{2} \delta^{3}}{r^{4}}\|u\|_{V}^{2}, \\
\left\|u_{3}(\cdot, \cdot, \delta)-u_{3}(\cdot, \cdot, 0)\right\|_{L^{2}\left(\widehat{\omega}_{\varepsilon}\right)}^{2} \leq C \varepsilon\|\nabla u\|_{L^{2}\left(\Omega_{\delta}^{+}\right)}^{2}+C \frac{\varepsilon^{2} \delta}{r^{2}}\|u\|_{V}^{2}
\end{gathered}
$$

A proof of the lemma is shifted to Appendix A.

\subsubsection{Estimates on displacements in $\Omega_{\delta}^{+}$}

Lemma 3.4. There exists a constant $C$ which does not depend on $\varepsilon, r$ and $\delta$, such that for any $u \in \mathbb{V}$

$$
\begin{gathered}
\left\|u_{\alpha}\right\|_{H^{1}\left(\Omega_{\delta}^{+}\right)} \leq C \frac{\varepsilon \delta^{3 / 2}}{r^{2}}\|u\|_{V}+C\|u\|_{V}, \\
\left\|u_{3}\right\|_{H^{1}\left(\Omega_{\delta}^{+}\right)} \leq C \frac{\varepsilon^{3 / 2} \delta^{3 / 2}}{r^{2}}\|u\|_{V}+C\|u\|_{V},
\end{gathered}
$$

where $\alpha=1,2$.

A proof of the lemma is shifted to Appendix A.

As a consequence of Lemma 3.4 and estimate (3.11), the inequality (3.12) can be replaced by

$$
\begin{gathered}
\left\|u_{\alpha}(\cdot, \cdot, \delta)-u_{\alpha}(\cdot, \cdot, 0)\right\|_{L^{2}\left(\widehat{\omega}_{\varepsilon}\right)}^{2} \leq C \frac{\varepsilon^{2} \delta^{3}}{r^{4}}\|u\|_{V}^{2} \\
\left\|u_{3}(\cdot, \cdot, \delta)-u_{3}(\cdot, \cdot, 0)\right\|_{L^{2}\left(\widehat{\omega}_{\varepsilon}\right)}^{2} \leq C \frac{\varepsilon^{3} \delta^{3}}{r^{4}}\|u\|_{V}^{2}+C \frac{\varepsilon^{2} \delta}{r^{2}}\|u\|_{V}^{2} .
\end{gathered}
$$

\subsubsection{Estimates for the set of beams $\Omega_{r, \varepsilon, \delta}^{i}$}

Lemma 3.5. There exists a constant $C$ which does not depend on $\varepsilon, r$ and $\delta$, such that for any $u \in \mathbb{V}$

$$
\begin{aligned}
\|\nabla u\|_{L^{2}\left(\Omega_{r, \varepsilon, \delta}^{i}\right)} & \leq C \frac{\delta}{r}\|u\|_{V}, \\
\left\|u_{3}\right\|_{L^{2}\left(\Omega_{r, \varepsilon, \delta}^{i}\right)} & \leq C \frac{r \delta^{1 / 2}}{\varepsilon}\|u\|_{V}, \\
\left\|u_{\alpha}\right\|_{L^{2}\left(\Omega_{r, \varepsilon, \delta}^{i}\right)} & \leq C \frac{r \delta^{1 / 2}}{\varepsilon}\left(1+\frac{\varepsilon \delta^{3 / 2}}{r^{2}}\right)\|u\|_{V},
\end{aligned}
$$

where $\alpha=1,2$.

A proof can be found in Appendix A 


\subsection{The limit cases}

In view of the estimates of Lemma 3.4 and in order to guarantee that the lower and upper parts of our structure match, we must assume that

$$
\frac{\varepsilon^{2} \delta^{3}}{r^{4}} \text { is uniformly bounded from above. }
$$

From now on, the parameters $r, \delta$ and $\varepsilon$ are linked in the following way

- $r=\kappa_{0} \varepsilon^{\eta_{0}}, \eta_{0} \geq 1, \kappa_{0}>0$, if $\eta_{0}=1$ then $\kappa_{0} \in(0,1 / 2)$ (limiting case: solid layer without holes),

- $\delta=\kappa_{1} \varepsilon^{\eta_{1}}, \kappa_{1}>0$ and $\eta_{1} \geq \eta_{0}$, (in order to deal with the beams).

The assumption (3.18) above yields

$$
2+3 \eta_{1}-4 \eta_{0} \geq 0
$$

Hence we distinguish three important cases:

- (i) $r=\kappa_{0} \varepsilon, \kappa_{0} \in(0,1 / 2)$ and $\delta=\kappa_{1} \varepsilon^{2 / 3}, \kappa_{1}>0$,

- (ii) $r=\kappa_{0} \varepsilon^{\eta_{0}}, \eta_{0} \in(1,2), \kappa_{0}>0$ and $\delta=\kappa_{1} \varepsilon^{\left(4 \eta_{0}-2\right) / 3}, \kappa_{1}>0$,

- (iii) $r=\kappa_{0} \varepsilon^{2}, \kappa_{0}>0$ and $\delta=\kappa_{1} \varepsilon^{2}, \kappa_{1}>0$.

For a sake of simplicity, we will use the following notations from now on:

- $\Omega_{\varepsilon}$ instead of $\Omega_{r, \varepsilon, \delta}$,

- $\Omega_{\varepsilon}^{i}$ instead of $\Omega_{r, \varepsilon, \delta}^{i}$,

- $\Omega_{\varepsilon}^{+}$instead of $\Omega_{\delta}^{+}$,

- $\sigma_{\varepsilon}$ instead of $\sigma_{r, \varepsilon, \delta}$,

- $u_{\varepsilon}$ instead of $u_{r, \varepsilon, \delta}$,

- $f_{\varepsilon}$ instead of $f_{r, \varepsilon, \delta}$.

With assumption (3.18) we can rewrite the estimates obtained above. For any $u \in \mathbb{V}$ we have

$$
\begin{gathered}
\|u\|_{L^{2}\left(\Omega_{\varepsilon}^{i}\right)} \leq C \frac{r \delta^{1 / 2}}{\varepsilon}\|u\|_{V}, \\
\|u\|_{H^{1}\left(\Omega_{\varepsilon}^{+}\right)} \leq C\|u\|_{V} .
\end{gathered}
$$

The constants do not depend on $\varepsilon, r$ and $\delta$.

\subsection{Force assumptions and the final a priori estimate}

We set

$$
B_{1}=D_{1} \times(0,1) .
$$

To obtain estimates on $u_{\varepsilon}$ we test $(2.9)$ with $\varphi=u_{\varepsilon}$. We have

$$
M_{1}\left\|u_{\varepsilon}\right\|_{V}^{2} \leq\left\|f_{\varepsilon}\right\|_{L^{2}\left(\Omega_{\varepsilon}, \mathbb{R}^{3}\right)}\left\|u_{\varepsilon}\right\|_{L^{2}\left(\Omega_{\varepsilon}, \mathbb{R}^{3}\right)} .
$$

We consider the following assumption on the applied forces:

$$
f_{\varepsilon}(x)= \begin{cases}\frac{\varepsilon^{2}}{r^{2} \delta} F^{m}\left(\varepsilon\left[\frac{x^{\prime}}{\varepsilon}\right]_{Y}, \frac{\varepsilon}{r}\left\{\frac{x^{\prime}}{\varepsilon}\right\}_{Y}, \frac{x_{3}}{\delta}\right) & \text { for a.e. } x \in \Omega_{\varepsilon}^{i}, \\ F(x) & \text { for a.e. } x \in \Omega^{-} \cup \Omega_{\varepsilon}^{+},\end{cases}
$$

where $F^{m} \in \mathcal{C}^{0}\left(\bar{\omega}, L^{2}\left(B_{1}, \mathbb{R}^{3}\right)\right), F \in L^{2}\left(\omega \times(-L, L), \mathbb{R}^{3}\right)$. Then,

$$
\left\|f_{\varepsilon}\right\|_{L^{2}\left(\Omega_{\varepsilon}^{i}, \mathbb{R}^{3}\right)} \leq \frac{\varepsilon}{r \delta^{1 / 2}}\left\|F^{m}\right\|_{L^{\infty}\left(\omega, L^{2}\left(B_{1}, \mathbb{R}^{3}\right)\right)} .
$$

Making use of the estimates (2.11), (3.19), (3.20) together with the inequality (3.21) yields

$$
\left\|u_{\varepsilon}\right\|_{V} \leq C .
$$

The constant $C$ does not depend of $r, \varepsilon$ and $\delta$.

From now on, we only consider cases (i) and (ii) introduced in Section 3.3. 


\section{Periodic unfolding operators. Taking the limit}

Definition 4.1. For a Lebesgue-measurable function $\varphi$ on $\omega \times(0, \delta)$, the unfolding operator $\mathcal{T}_{\varepsilon}$ is defined as follows:

$$
\mathcal{T}_{\varepsilon}(\varphi)\left(s_{1}, s_{2}, X_{3}\right)=\left\{\begin{array}{lll}
\varphi\left(s_{1}, s_{2}, \delta X_{3}\right), & \text { for a.e. } & \left(s_{1}, s_{2}, X_{3}\right) \in \widehat{\omega}_{\varepsilon} \times(0,1), \\
0, & \text { for a.e. } & \left(s_{1}, s_{2}, X_{3}\right) \in \Lambda_{\varepsilon} \times(0,1) .
\end{array}\right.
$$

Definition 4.2. For a Lebesgue-measurable function $\varphi$ on $\omega \times B_{r, \delta}$, the unfolding operator $\mathcal{T}_{\varepsilon}^{\prime}$ is defined as follows:

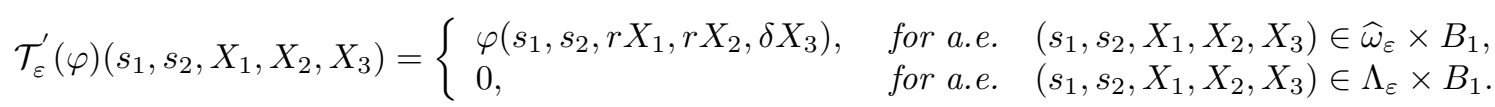

The first two coordinates in the operators above deal with the macro-position of a point in a periodic layer, whereas the others are responsible for the position in a unit cell.

The properties of these unfolding operators are proved in Lemma B.1 in Appendix B.

\subsection{The limit fields. Cases (i) and (ii)}

From now on, $\left(u_{\varepsilon}\right)_{\alpha}$ will be denoted as $u_{\varepsilon, \alpha}$; the same notation will be used for the fields with values in $\mathbb{R}^{2}$ or $\mathbb{R}^{3}$.

The compactness results are given in Appendix B, Lemma B.2.

Further we extend the function $u_{\varepsilon}$ defined on the domain $\Omega_{\varepsilon}^{+}$by reflection to the domain $\omega \times(\delta, L+\delta)$. The new function is also denoted $u_{\varepsilon}$.

Proposition 4.1. There exist a subsequence of $\{\varepsilon\}$, still denoted by $\{\varepsilon\}$, and $u^{ \pm} \in H^{1}\left(\Omega^{ \pm}, \mathbb{R}^{3}\right)$ with $u^{-}=$ 0 on $\Gamma$ and $\widetilde{\mathcal{R}} \in L^{2}\left(\omega, H_{0}^{1}\left((0,1), \mathbb{R}^{3}\right)\right), \widetilde{\mathcal{U}}_{\alpha} \in L^{2}\left(\omega, H^{2}(0,1)\right), \widetilde{\mathcal{U}}_{3}, \quad \widetilde{\mathcal{U}}_{3}^{\prime} \in L^{2}\left(\omega, H^{1}(0,1)\right), \widetilde{u} \in L^{2}(\omega \times$ $\left.(0,1), H^{1}\left(D_{1}, \mathbb{R}^{3}\right)\right)$ and $Z \in L^{2}\left(\omega \times(0,1), \mathbb{R}^{3}\right)$ such that

$$
\begin{gathered}
u_{\varepsilon} \rightarrow u^{-} \quad \text { weakly in } H^{1}\left(\Omega^{-}\right), \text {strongly in } L^{2}\left(\Omega^{-}\right), \\
u_{\varepsilon}\left(\cdot+\delta \mathbf{e}_{3}\right) \rightarrow u^{+} \quad \text { weakly in } H^{1}\left(\Omega^{+}\right), \text {strongly in } L^{2}\left(\Omega^{+}\right),
\end{gathered}
$$

$$
\begin{gathered}
\delta \mathcal{T}_{\varepsilon}\left(\widetilde{\mathcal{R}}_{\varepsilon}\right) \rightarrow \widetilde{\mathcal{R}} \quad \text { weakly in } L^{2}\left(\omega, H^{1}(0,1)\right) \text {, such that } \\
\widetilde{\mathcal{R}}\left(x^{\prime}, 0\right)=\widetilde{\mathcal{R}}\left(x^{\prime}, 1\right)=0, \text { for a.e. } x^{\prime} \in \omega,
\end{gathered}
$$

$$
\begin{gathered}
\frac{\delta}{r}\left(\mathcal{T}_{\varepsilon}\left(\widetilde{\mathcal{U}}_{\varepsilon, 3}\right)-\widetilde{\mathcal{U}}_{\varepsilon, 3}(\cdot, \cdot, 0)\right) \rightarrow \widetilde{\mathcal{U}}_{3}^{\prime} \quad \text { weakly in } L^{2}\left(\omega, H^{1}(0,1)\right), \\
\mathcal{T}_{\varepsilon}\left(\widetilde{\mathcal{U}}_{\varepsilon, 3}\right) \rightarrow \widetilde{\mathcal{U}}_{3} \quad \text { weakly in } L^{2}\left(\omega, H^{1}(0,1)\right) \text {, such that } \\
\widetilde{\mathcal{U}}_{3}(\cdot, \cdot, \cdot)=\widetilde{\mathcal{U}}_{3}(\cdot, \cdot, 0)=u_{3 \mid \Sigma}^{-}=\widetilde{\mathcal{U}}_{3}(\cdot, \cdot, 1)=u_{3 \mid \Sigma}^{+}, \text {a.e. in } \omega \times(0,1), \\
\mathcal{T}_{\varepsilon}\left(\widetilde{\mathcal{U}}_{\varepsilon, \alpha}\right) \rightarrow \widetilde{\mathcal{U}}_{\alpha} \quad \text { weakly in } L^{2}\left(\omega, H^{1}(0,1)\right), \quad \text { for } \alpha=\overline{1,2} \text {, such that } \\
\widetilde{\mathcal{U}}_{\alpha}(\cdot, \cdot, 0)=u_{\alpha \mid \Sigma}^{-}, \quad \widetilde{\mathcal{U}}_{\alpha}(\cdot, \cdot, 1)=u_{\alpha \mid \Sigma}^{+} \text {a.e. in } \omega, \\
\frac{\partial \widetilde{\mathcal{U}}_{\alpha}}{\partial X_{3}}(\cdot, \cdot, 0)=\frac{\partial \widetilde{\mathcal{U}}_{\alpha}}{\partial X_{3}}(\cdot, \cdot, 1)=0 \text { a.e. in } \omega, \\
\frac{\partial \widetilde{\mathcal{U}}_{1}}{\partial X_{3}}=\widetilde{\mathcal{R}}_{2}, \quad \frac{\partial \widetilde{\mathcal{U}}_{2}}{\partial X_{3}}=-\widetilde{\mathcal{R}}_{1} \text { a.e. in } \omega \times(0,1), \\
\frac{\delta^{2}}{r^{2}} \mathcal{T}_{\varepsilon}^{\prime}\left(\widetilde{u}_{\varepsilon}\right) \rightarrow \widetilde{u} \quad \text { weakly in } L^{2}\left(\omega \times(0,1), H^{1}\left(D_{1}\right)\right), \\
\frac{\delta}{r} \mathcal{T}_{\varepsilon}^{\prime}\left(\widetilde{u}_{\varepsilon}\right) \rightarrow 0 \quad \text { weakly in } L^{2}\left(\omega, H^{1}\left(B_{1}\right)\right), \\
\left.\frac{\partial \mathcal{T}_{\varepsilon}\left(\widetilde{\mathcal{U}}_{\varepsilon}\right)}{\partial X_{3}}-\delta \mathcal{T}_{\varepsilon}\left(\widetilde{\mathcal{R}}_{\varepsilon} \wedge \mathbf{e}_{3}\right)\right) \rightarrow Z \\
\text { weakly in } L^{2}(\omega \times(0,1)) .
\end{gathered}
$$


The proof of the proposition is shifted to Appendix, Sec. B.

As an immediate consequence of Proposition 4.1, we have the following lemma.

Lemma 4.1. There exist a symmetric matrix field $X \in L^{2}\left(\omega \times B_{1}, \mathbb{R}^{9}\right)$ and a field $\widetilde{u^{\prime}} \in L^{2}\left(\omega \times(0,1), H^{1}\left(D_{1}, \mathbb{R}^{3}\right)\right)$, such that

$$
\frac{\delta^{2}}{r} \mathcal{T}_{\varepsilon}^{\prime}\left(\widetilde{e\left(u_{\varepsilon}\right)}\right) \rightarrow X \quad \text { weakly in } L^{2}\left(\omega \times B_{1}, \mathbb{R}^{9}\right),
$$

where $X$ is defined by

$$
\begin{aligned}
& X_{i j}=\frac{1}{2}\left(\frac{\partial \widetilde{u}_{i}^{\prime}}{\partial X_{j}}+\frac{\partial \widetilde{u}_{j}^{\prime}}{\partial X_{i}}\right), \quad i, j=1,2, \\
& X_{13}=X_{31}=\frac{1}{2}\left(\frac{\partial \widetilde{u}_{3}^{\prime}}{\partial X_{1}}-\frac{\partial \widetilde{\mathcal{R}}_{3}}{\partial X_{3}} X_{2}\right), \\
& X_{23}=X_{32}=\frac{1}{2}\left(\frac{\partial \widetilde{u}_{3}^{\prime}}{\partial X_{2}}+\frac{\partial \widetilde{\mathcal{R}}_{3}}{\partial X_{3}} X_{1}\right), \\
& X_{33}=\frac{\partial \widetilde{\mathcal{U}}_{3}^{\prime}}{\partial X_{3}}-\frac{\partial^{2} \widetilde{\mathcal{U}}_{2}}{\partial X_{3}^{2}} X_{2}-\frac{\partial^{2} \widetilde{\mathcal{U}}_{1}}{\partial X_{3}^{2}} X_{1} .
\end{aligned}
$$

Denote by $\Theta$ the weak limit of the unfolded stress tensor $\frac{\delta^{2}}{r} \mathcal{T}_{\varepsilon}^{\prime}\left(\sigma_{\varepsilon}\right)$ in $L^{2}\left(\omega \times B_{1}, \mathbb{R}^{9}\right)$ :

$$
\frac{\delta^{2}}{r} \mathcal{T}_{\varepsilon}^{\prime}\left(\sigma_{\varepsilon}\right) \rightarrow \Theta, \quad \text { weakly in } L^{2}\left(\omega \times B_{1}, \mathbb{R}^{9}\right) .
$$

Proceeding exactly as in Section 6.1 of [2] and Section 8.1 of [3], we first derive $\widetilde{u}^{\prime}$

$$
\begin{aligned}
& {\widetilde{u^{\prime}}}_{1}=\nu^{m}\left(-X_{1} \frac{\partial \widetilde{\mathcal{U}}_{3}^{\prime}}{\partial X_{3}}+\frac{X_{1}^{2}-X_{2}^{2}}{2} \frac{\partial^{2} \widetilde{\mathcal{U}}_{1}}{\partial X_{3}^{2}}+X_{1} X_{2} \frac{\partial^{2} \widetilde{\mathcal{U}}_{2}}{\partial X_{3}^{2}}\right), \\
& \widetilde{u^{\prime}}{ }_{2}=\nu^{m}\left(-X_{2} \frac{\partial \widetilde{\mathcal{U}}_{3}^{\prime}}{\partial X_{3}}+X_{1} X_{2} \frac{\partial^{2} \widetilde{\mathcal{U}}_{1}}{\partial X_{3}^{2}}+\frac{X_{2}^{2}-X_{1}^{2}}{2} \frac{\partial^{2} \widetilde{\mathcal{U}}_{2}}{\partial X_{3}^{2}}\right) .
\end{aligned}
$$

Similarly, the same computations as in Section 6.1 of [2] lead to $\widetilde{u^{\prime}}{ }_{3}=0$.

As a consequence of Lemma 4.1 we obtain

$$
\begin{aligned}
& \Theta_{11}=\Theta_{22}=\Theta_{12}=0, \\
& \Theta_{13}=-\mu^{m} X_{2} \frac{\partial \widetilde{\mathcal{R}}_{3}}{\partial X_{3}}, \quad \Theta_{23}=\mu^{m} X_{1} \frac{\partial \widetilde{\mathcal{R}}_{3}}{\partial X_{3}}, \\
& \Theta_{33}=E^{m}\left(\frac{\partial \widetilde{\mathcal{U}}_{3}^{\prime}}{\partial X_{3}}-X_{1} \frac{\partial^{2} \widetilde{\mathcal{U}}_{1}}{\partial X_{3}^{2}}-X_{2} \frac{\partial^{2} \widetilde{\mathcal{U}}_{2}}{\partial X_{3}^{2}}\right) .
\end{aligned}
$$

\section{The limit problem}

\subsection{The equations for the domain $\Omega_{\varepsilon}^{i}$}

Proposition 5.1. $\left(\widetilde{\mathcal{U}}_{1}, \widetilde{\mathcal{U}}_{2}\right)$ satisfy the variational formulation

$$
\begin{array}{r}
\frac{\pi \kappa_{0}^{4}}{4 \kappa_{1}^{3}} E^{m} \int_{0}^{1} \frac{\partial^{2} \widetilde{\mathcal{U}}_{\alpha}}{\partial X_{3}^{2}}\left(x^{\prime}, X_{3}\right) \frac{d^{2} \varphi_{\alpha}}{d X_{3}^{2}}\left(X_{3}\right) d X_{3}=\int_{0}^{1} \widetilde{F}_{\alpha}^{m}\left(x^{\prime}, X_{3}\right) \varphi_{\alpha}\left(X_{3}\right) d X_{3}, \\
\forall \varphi_{\alpha} \in H_{0}^{2}(0,1), \quad \text { for a.e. } x^{\prime} \in \omega,
\end{array}
$$

where

$$
\widetilde{F}_{\alpha}^{m}\left(x^{\prime}, X_{3}\right)=\int_{D_{1}} F_{\alpha}^{m}\left(x^{\prime}, X\right) d X_{1} d X_{2} \quad \text { a.e. in } \omega \times(0,1) \quad \alpha=1,2 .
$$

Furthermore, $\widetilde{\mathcal{R}}_{3}=0$, and there exists $a \in L^{2}(\omega)$ such that

$$
\widetilde{\mathcal{U}}_{3}^{\prime}\left(x^{\prime}, X_{3}\right)=a\left(x^{\prime}\right) X_{3} \quad \text { a.e. in } \omega \times(0,1) .
$$

The detailed proof of this Proposition and all main results can be found in Appendix C. 


\subsection{The equations for the macroscopic domain}

Denote

$$
\begin{aligned}
& \mathbb{V}=\left\{v \in L^{2}\left(\Omega^{-} \cup \Omega^{+} ; \mathbb{R}^{3}\right) \mid v_{\mid \Omega^{-}} \in H^{1}\left(\Omega^{-} ; \mathbb{R}^{3}\right) \text { and } v_{\mid \Omega^{-}}=0 \text { on } \Gamma\right. \\
& \left.v_{\mid \Omega^{+}} \in H^{1}\left(\Omega^{+} ; \mathbb{R}^{3}\right) \text { and } v_{3 \mid \Omega^{+}}=v_{3 \mid \Omega^{-}} \text {on } \Sigma\right\} \\
& \mathbb{V}_{T}=\left\{\left(v, \mathcal{V}_{1}, \mathcal{V}_{2}, \mathcal{V}_{3}, \mathcal{V}_{4}\right) \in \mathbb{V} \times\left[L^{2}\left(\Omega ; H^{2}(0,1)\right)\right]^{2} \times\left[L^{2}\left(\Omega ; H^{1}(0,1)\right)\right]^{2} \mid\right. \\
& \mathcal{V}_{\alpha}(\cdot, \cdot, 0)=v_{\alpha \mid \Sigma}^{-}, \quad \mathcal{V}_{\alpha}(\cdot, \cdot, 1)=v_{\alpha \mid \Sigma}^{+} \text {a.e. in } \omega, \\
& \left.\mathcal{V}_{3}(\cdot, \cdot, 0)=\mathcal{V}_{4}(\cdot, \cdot, 0)=\mathcal{V}_{4}(\cdot, \cdot, 1)=\frac{\partial \mathcal{V}_{\alpha}}{\partial X_{3}}(\cdot, \cdot, 0)=\frac{\partial \mathcal{V}_{\alpha}}{\partial X_{3}}(\cdot, \cdot, 1)=0 \text { a.e. in } \omega, \quad \alpha \in\{1,2\}\right\}
\end{aligned}
$$

Let $\chi$ be in $\mathcal{C}_{c}^{\infty}\left(\mathbb{R}^{2}\right)$ such that $\chi(y)=1$ in $D_{1} . D_{1}$ is the disc centered in $O=(0,0)$ with radius 1 .

From now on we only consider the case (ii).

\subsubsection{Determination of $\widetilde{\mathcal{U}}_{3}^{\prime}$}

Let $\chi$ be in $\mathcal{C}_{c}^{\infty}\left(\mathbb{R}^{2}\right)$ such that $\chi(y)=1$ in $D_{1}$.

Lemma 5.1. Let $\phi$ be in $W^{1, \infty}(\omega)$, and let $\phi_{\varepsilon, r}$ be defined by

$$
\phi_{\varepsilon, r}\left(x^{\prime}\right)=\chi\left(\frac{\varepsilon}{r}\left\{\frac{x^{\prime}}{\varepsilon}\right\}_{Y}\right) \phi\left(\varepsilon\left[\frac{x^{\prime}}{\varepsilon}\right]_{Y}\right)+\left[1-\chi\left(\frac{\varepsilon}{r}\left\{\frac{x^{\prime}}{\varepsilon}\right\}_{Y}\right)\right] \phi\left(x^{\prime}\right) \quad \text { for a.e. } x^{\prime} \in \omega .
$$

If $\frac{r}{\varepsilon} \rightarrow 0$, then for every $p \in[1,+\infty)$ we have

$$
\phi_{\varepsilon, r} \longrightarrow \phi \quad \text { strongly in } W^{1, p}(\omega) .
$$

See in Appendix C for the proof.

Lemma 5.2. The function a introduced in Proposition 5.1 is equal to 0 and

$$
\tilde{\mathcal{U}}_{3}^{\prime}\left(x^{\prime}, X_{3}\right)=0 \quad \text { a.e. in } \omega \times(0,1) .
$$

This Lemma is proved in Appendix $\mathrm{C}$ as well.

As a consequence of the lemma above and Proposition 5.1, one gets

$$
\begin{aligned}
& \Theta_{i j}=0, \quad(i, j) \neq(3,3) \\
& \Theta_{33}=-E^{m}\left(X_{1} \frac{\partial^{2} \widetilde{\mathcal{U}}_{1}}{\partial X_{3}^{2}}+X_{2} \frac{\partial^{2} \widetilde{\mathcal{U}}_{2}}{\partial X_{3}^{2}}\right) .
\end{aligned}
$$

\subsubsection{Determination of $u_{\alpha}^{ \pm}$and $u_{3}$}

Theorem 5.1. The variational formulation of the limit problem for (2.9) is

$$
\begin{gathered}
\int_{\Omega^{+} \cup \Omega^{-}} \sigma^{ \pm}: e(v) d x+\frac{\pi \kappa_{0}^{4}}{4 \kappa_{1}^{3}} E^{m} \int_{\omega \times(0,1)} \sum_{\alpha=1}^{2} \frac{\partial^{2} \widetilde{\mathcal{U}}_{\alpha}}{\partial X_{3}^{2}} \frac{\partial^{2} \psi_{\alpha}}{\partial X_{3}^{2}} d x^{\prime} d X_{3} \\
+\frac{\pi \kappa_{0}^{4}}{4 \kappa_{1}^{3}} \mu^{m} \int_{\omega \times(0,1)} \frac{\partial \widetilde{\mathcal{R}}_{3}}{\partial X_{3}} \frac{\partial \psi_{4}}{\partial X_{3}} d x^{\prime} d X_{3}+\frac{\pi \kappa_{0}^{4}}{\kappa_{1}^{3}} E^{m} \int_{\omega \times(0,1)} \frac{\partial \widetilde{\mathcal{U}}_{3}^{\prime}}{\partial X_{3}} \frac{\partial \Phi_{3}}{\partial X_{3}} d x^{\prime} d X_{3} \\
=\int_{\Omega^{+} \cup \Omega^{-}} F v d x+\int_{\omega \times(0,1)} \sum_{\alpha=1}^{2} \widetilde{F}_{\alpha}^{m} \psi_{\alpha} d x^{\prime} d X_{3}+\int_{\omega} \bar{F}_{3}^{m} v_{3} d x^{\prime}, \\
\forall\left(v, \psi_{1}, \psi_{2}, \psi_{3}, \psi_{4}\right) \in \mathbb{V}_{T}, \quad \forall \Phi_{3} \in L^{2}\left(\omega ; H_{0}^{1}(0,1)\right),
\end{gathered}
$$

where

$$
\bar{F}_{3}^{m}\left(x^{\prime}\right)=\int_{B_{1}} F_{3}^{m}\left(x^{\prime}, X\right) d X, \quad x^{\prime} \in \omega .
$$

We refer to Appendix $\mathrm{C}$ for the proof.

We also send the reader to Sec.C.2.3 of the Appendix, for the case $(i)$. 


\subsection{The strong formulation of the limit problem}

The strong formulations are the same for both cases $(i),(i i)$. We will use the following notation.

Notation 5.1. The convolution of functions $K$ and $F$ is

$$
\left(K * \widetilde{F}_{\alpha}^{m}\right)\left(x^{\prime}, X_{3}\right)=\int_{0}^{1} K\left(X_{3}, y_{3}\right) \widetilde{F}_{\alpha}^{m}\left(x^{\prime}, y_{3}\right) d y_{3}
$$

Define

$$
\begin{gathered}
\sigma^{ \pm}=\lambda^{b}\left(\operatorname{Tr} e\left(u^{ \pm}\right)\right) I+2 \mu^{b} e\left(u^{ \pm}\right) \in L^{2}\left(\Omega^{ \pm} ; \mathbb{R}^{9}\right), \\
K_{\alpha}\left(X_{3}, y_{3}\right)=D\left(X_{3}-y_{3}\right) X_{3}^{2}\left(3-2 X_{3}\right)+6\left(1-2 X_{3}\right)\left(\left(X_{3}-y_{3}\right) H\left(X_{3}-y_{3}\right)+\left(1-y_{3}\right)^{2}\left(y_{3}-2 y_{3} X_{3}-X_{3}\right)\right)
\end{gathered}
$$

where $H(x)$ is the Heaviside function and $D(x)$ the Dirac delta distribution at point $x$.

Let $\{\varepsilon\}$ be a sequence of positive real numbers which tends to 0 . Let $\left(u_{\varepsilon}, \sigma_{\varepsilon}\right)$ be the solution of $(2.9)$ and $\widetilde{\mathcal{U}}_{\varepsilon}$ and $\widetilde{\mathcal{R}}_{\varepsilon}$ be the first two terms of the decompostion of $u_{\varepsilon}$ on $\Omega_{\varepsilon}^{i}$. Let $f$ satisfy assumptions $(3.22)$. Then the limit problems for cases $(i),(i i)$ can be written as follows.

Bending problem in the beams: $\left(\widetilde{\mathcal{U}}_{1}, \widetilde{\mathcal{U}}_{2}\right) \in L^{2}\left(\omega, H^{1}(0,1)\right)^{2}$ is the unique solution of the problem

$$
\left\{\begin{array}{l}
\frac{\pi \kappa_{0}^{4}}{4 \kappa_{1}^{3}} E^{m} \frac{\partial^{4} \widetilde{\mathcal{U}}_{\alpha}}{\partial X_{3}^{4}}=\widetilde{F}_{\alpha}^{m} \quad \text { a.e. in } \omega \times(0,1) \\
\frac{\partial \widetilde{\mathcal{U}}_{\alpha}}{\partial X_{3}}(\cdot, \cdot, 0)=\frac{\partial \widetilde{\mathcal{U}}_{\alpha}}{\partial X_{3}}(\cdot, \cdot, 1)=0, \quad \text { a.e. in } \omega \\
\widetilde{\mathcal{U}}_{\alpha}(\cdot, \cdot, 0)=u_{\alpha \mid \Sigma}^{-}, \quad \widetilde{\mathcal{U}}_{\alpha}(\cdot, \cdot, 1)=u_{\alpha \mid \Sigma}^{+}, \quad \text { a.e. in } \omega
\end{array}\right.
$$

3D elasticity problem in $\Omega^{+} \cup \Omega^{-}:\left(u^{ \pm}, \sigma^{ \pm}\right) \in\left(H^{1}\left(\Omega^{+} \cup \Omega^{-}\right)\right)^{3} \times\left(L^{2}(\Omega)\right)_{S}^{3 \times 3}$ is the unique weak solution of the problem

$$
-\sum_{j=1}^{3} \frac{\partial \sigma_{i j}^{ \pm}}{\partial x_{j}}=F_{i} \quad \text { in } \Omega^{ \pm}, \quad i=1,2,3
$$

together with the boundary conditions

$$
\begin{cases}\sigma_{i 3}^{+}=0 & \text { in } \omega \times\{L\} \\ \sigma_{i 3}^{-}=0 & \text { in } \omega \times\{-L\}\end{cases}
$$

and the transmission conditions

$$
\begin{cases}{\left[\sigma_{i 3}^{ \pm}\right]_{\mid \Sigma}=\bar{F}_{i}^{m}} & \text { on } \Sigma \\ {\left[u_{3}^{ \pm}\right]_{\mid \Sigma}=0} & \text { on } \Sigma, \\ \sigma_{\alpha 3}^{+}=-\frac{3 \pi \kappa_{0}^{4}}{\kappa_{1}^{3}} E^{m}\left[u_{\alpha}^{ \pm}\right]_{\mid \Sigma}+\int_{0}^{1} K_{\alpha} * \widetilde{F}_{\alpha}^{m} d X_{3} & \text { on } \Sigma, \quad \alpha=1,2 .\end{cases}
$$

\subsection{The weak formulation of the limit problem}

Lemma 5.3. The weak formulation of the limit problem can be rewritten as

$$
\begin{aligned}
\int_{\Omega^{+} \cup \Omega^{-}} \sigma^{ \pm}: e(v) & d x+\frac{3 \pi \kappa_{0}^{4}}{\kappa_{1}^{3}} E^{m} \int_{\Sigma} \sum_{\alpha=1}^{2}\left[u_{\alpha}^{ \pm}\right]_{\mid \Sigma}\left[v_{\alpha}^{ \pm}\right]_{\mid \Sigma} d s= \\
= & \int_{\Omega^{+} \cup \Omega^{-}} F v d x+\int_{\Sigma} \sum_{\alpha=1}^{3} \bar{F}_{\alpha}^{m} v_{\alpha}^{-} d s+\int_{\Sigma} \sum_{\alpha=1}^{2}\left[v_{\alpha}^{ \pm}\right]_{\mid \Sigma} \int_{0}^{1} K_{\alpha} * \widetilde{F}_{\alpha}^{m} d X_{3} d s, \quad \forall v \in \mathbb{V}
\end{aligned}
$$

Proof. Step 1. Decomposition of $\widetilde{\mathcal{U}}_{\alpha}$.

Denote

$$
\mathcal{V}_{d}=\left\{\eta \in \mathcal{C}^{3}([0,1]) \mid \eta\left(X_{3}\right)=(b-a) X_{3}^{2}\left(3-2 X_{3}\right)+a, \quad(a, b) \in \mathbb{R}^{2}\right\} .
$$

Observe that a function $X_{3} \longmapsto \eta\left(X_{3}\right)=(b-a) X_{3}^{2}\left(3-2 X_{3}\right)+a$ of $\mathcal{V}_{d}$ satisfies

$$
\eta(0)=a, \quad \eta(1)=b, \quad \frac{d \eta}{d X_{3}}(0)=0, \quad \frac{d \eta}{d X_{3}}(1)=0, \quad \text { and } \quad \frac{d^{4} \eta}{d X_{3}^{4}}=0 \text { in }(0,1) .
$$


Hence for any function $\psi \in H_{0}^{2}(0,1)$ we have

$$
\int_{0}^{1} \frac{d^{2} \eta}{d X_{3}^{2}}(t) \frac{d^{2} \psi}{d X_{3}^{2}}(t) d t=0
$$

Let $\widehat{\mathcal{U}}_{\alpha}$ be in $L^{2}\left(\omega ; H_{0}^{2}(0,1)\right)$ the solution of the following problem:

$$
\left\{\begin{array}{l}
\frac{\pi \kappa_{0}^{4}}{4 \kappa_{1}^{3}} E^{m} \frac{\partial^{4} \widehat{\mathcal{U}}_{\alpha}}{\partial X_{3}^{4}}\left(x^{\prime}, X_{3}\right)=\widetilde{F}_{\alpha}^{m}\left(x^{\prime}, X_{3}\right) \quad \text { a.e. in } \omega \times(0,1) \\
\frac{\partial \widehat{\mathcal{U}}_{\alpha}}{\partial X_{3}}(\cdot, \cdot, 0)=\frac{\partial \widehat{\mathcal{U}}_{\alpha}}{\partial X_{3}}(\cdot, \cdot, 1)=0, \quad \text { a.e. in } \omega \\
\widehat{\mathcal{U}}_{\alpha}(\cdot, \cdot, 0)=\widehat{\mathcal{U}}_{\alpha}(\cdot, \cdot, 1)=0, \quad \text { a.e. in } \omega .
\end{array}\right.
$$

Using Green's function we can write $\widehat{\mathcal{U}}_{\alpha}$ in the following way:

$$
\widehat{\mathcal{U}}_{\alpha}\left(x^{\prime}, X_{3}\right)=\frac{4 \kappa_{1}^{3}}{\pi E^{m} \kappa_{0}^{4}} \int_{0}^{1} \xi_{\alpha}\left(X_{3}, y_{3}\right) \widetilde{F}_{\alpha}^{m}\left(x^{\prime}, y_{3}\right) d y_{3},
$$

where $\xi_{\alpha}$ is the solution of the equation

$$
\left\{\begin{array}{l}
\frac{d^{4} \xi_{\alpha}}{d X_{3}^{4}}=D\left(X_{3}-y_{3}\right), \quad y_{3} \in(0,1) \\
\frac{d \xi_{\alpha}}{d X_{3}}(0)=\frac{d \xi_{\alpha}}{d X_{3}}(1)=0 \\
\xi_{\alpha}(0)=\xi_{\alpha}(1)=0
\end{array}\right.
$$

Solving the equation above we obtain

$$
\xi_{\alpha}\left(X_{3}, y_{3}\right)=\frac{1}{6}\left(X_{3}-y_{3}\right)^{3} H\left(X_{3}-y_{3}\right)-\frac{1}{6}\left(1-y_{3}\right)^{2}\left(2 y_{3}+1\right) X_{3}^{3}+\frac{1}{2}\left(1-y_{3}\right)^{2} y_{3} X_{3}^{2}
$$

The function $\widetilde{\mathcal{U}}_{\alpha}$ is uniquely decomposed as a function belonging to $L^{2}\left(\omega ; \mathcal{V}_{d}\right)$ and a function in $L^{2}\left(\omega ; H_{0}^{2}(0,1)\right)$ :

$$
\begin{aligned}
\widetilde{\mathcal{U}}_{\alpha}\left(x^{\prime}, X_{3}\right) & =\left(1-X_{3}\right)^{2}\left(2 X_{3}+1\right) u_{\alpha \mid \Sigma}^{-}\left(x^{\prime}\right)-X_{3}^{2}\left(3-2 X_{3}\right) u_{\alpha \mid \Sigma}^{+}\left(x^{\prime}\right)+\widehat{\mathcal{U}}_{\alpha}\left(x^{\prime}, X_{3}\right) \\
& =\overline{\mathcal{U}}_{\alpha}\left(x^{\prime}, X_{3}\right)+\widehat{\mathcal{U}}_{\alpha}\left(x^{\prime}, X_{3}\right) \quad \text { for a.e. }\left(x^{\prime}, X_{3}\right) \in \omega \times(0,1) .
\end{aligned}
$$

Step 2. Taking into account the decomposition (5.10) and using as a test function $\psi_{\alpha}=\left[v_{\alpha}^{ \pm}\right]_{\mid \Sigma} X_{3}^{2}(3-$ $\left.2 X_{3}\right)+v_{\alpha \mid \Sigma}^{-}$in $(5.3)$ we obtain

$$
\begin{gathered}
\int_{\Omega^{+} \cup \Omega^{-}} \sigma^{ \pm}: e(v) d x+\frac{3 \pi \kappa_{0}^{4}}{2 \kappa_{1}^{3}} E^{m} \int_{\omega} \sum_{\alpha=1}^{2} \int_{0}^{1}\left(\frac{\partial^{2} \overline{\mathcal{U}}_{1}}{\partial X_{3}^{2}}\left[v_{1}^{ \pm}\right]_{\mid \Sigma}+\frac{\partial^{2} \overline{\mathcal{U}}_{2}}{\partial X_{3}^{2}}\left[v_{2}^{ \pm}\right]_{\mid \Sigma}\right)\left(1-2 X_{3}\right) d X_{3} d x^{\prime}= \\
=\int_{\Omega^{+} \cup \Omega^{-}} F v d x+\int_{\omega} \sum_{\alpha=1}^{2}\left[v_{\alpha}^{ \pm}\right]_{\mid \Sigma} \int_{0}^{1}\left(\widetilde{F}_{\alpha}^{m} X_{3}^{2}\left(3-2 X_{3}\right)-\frac{3 \pi \kappa_{0}^{4}}{2 \kappa_{1}^{3}} E^{m} \frac{\partial^{2} \widehat{\mathcal{U}}_{\alpha}}{\partial X_{3}^{2}}\left(1-2 X_{3}\right)\right) d X_{3} d x^{\prime}+ \\
+\int_{\omega} \sum_{\alpha=1}^{3} \bar{F}_{\alpha}^{m} v_{\alpha}^{-} d x^{\prime}
\end{gathered}
$$

Making use of the solutions for $\overline{\mathcal{U}}_{\alpha}$ and $\widehat{\mathcal{U}}_{\alpha}$ we can write

$$
\begin{aligned}
& \int_{\Omega^{+} \cup \Omega^{-}} \sigma^{ \pm}: e(v) d x+\frac{3 \pi \kappa_{0}^{4}}{\kappa_{1}^{3}} E^{m} \int_{\omega} \sum_{\alpha=1}^{2}\left[u_{\alpha}^{ \pm}\right]_{\mid \Sigma}\left[v_{\alpha}^{ \pm}\right]_{\mid \Sigma} d x^{\prime}=\int_{\Omega^{+} \cup \Omega^{-}} F v d x+ \\
& +\int_{\omega} \sum_{\alpha=1}^{2}\left[v_{\alpha}^{ \pm}\right]_{\mid \Sigma} \int_{0}^{1}\left(\widetilde{F}_{\alpha}^{m} X_{3}^{2}\left(3-2 X_{3}\right)-6\left(1-2 X_{3}\right) \int_{0}^{1} \frac{d^{2} \xi_{\alpha}}{d X_{3}^{2}}\left(X_{3}, y_{3}\right) \widetilde{F}_{\alpha}^{m}\left(x^{\prime}, y_{3}\right) d y_{3}\right) d X_{3} d x^{\prime}+ \\
& +\int_{\omega} \sum_{\alpha=1}^{3} \bar{F}_{\alpha}^{m} v_{\alpha}^{-} d x^{\prime}
\end{aligned}
$$

Using the notation for the convolution and the expression for $\frac{d^{2} \xi_{\alpha}}{d X_{3}^{2}}$ we get the result. 
From the variational formulation (5.8) the final strong formulation is obtained.

Remark 5.1. The limit model can have an in-plane discontinuity at the interface. This is the limit behavior resulting from the deformation of the beams in the thin layer, whose bending leads to localized shearing at the interface but which are basically inextensible in the transverse direction.

We note that starting with a linear elasticity for the blocks and the layer in-between, we end up with a kind of a linearized contact problem for two elastic blocks in the limit. A suitable choice of the load in the right-hand side should keep the tangential sliding small, such that the context of the linearity and small deformations is not violated.

\section{Additional remarks}

\subsection{Other possible configurations}

In this section we outline other possible configurations.

Remark 6.1. The case

$$
r=\kappa_{1} \varepsilon^{2}, \quad \delta=\kappa_{2} \varepsilon^{2}, \quad \kappa_{1}, \kappa_{2}>0,
$$

can also be considered, but should be studied separately. The structure obtained in this context will no longer correspond to the set of the thin beams but to some kind of a perforated domain.

Remark 6.2. For the case $\frac{\varepsilon^{2} \delta^{3}}{r^{4}} \rightarrow 0$, we obtain from the estimates (3.15), (3.16) that

$$
\lim _{r, \varepsilon, \delta \rightarrow 0}\|u(\cdot, \cdot, \delta)-u(\cdot, \cdot, 0)\|_{L^{2}\left(\widehat{\omega}_{\varepsilon}\right)}=0
$$

Therefore,

$$
u^{+}{ }_{\mid \Sigma}=u^{-}{ }_{\mid \Sigma}
$$

where $u^{ \pm} \in H^{1}\left(\Omega^{+} \cup \Omega^{-}, \Gamma\right)$ is the limit of the function $u_{\varepsilon}$. Hence we obtain two limit problems on the domains $\Omega^{+}, \Omega^{-}$with Dirichlet boundary conditions, and the layer has no influence on the limit problem.

\subsection{Numerical illustration}

In this section solutions $u_{r, \varepsilon, \delta}$ of the equation (2.8) are compared with the solution $u$ of $(5.5)-(5.7)$ for the $2 \mathrm{D}$ case. The solutions $u_{r, \varepsilon, \delta}$ are computed numerically for different $r, \varepsilon, \delta$ with the commercial finite element software COMSOL Multiphysics. Triangular elements were used for the computations. The relation between the parameters is chosen in a following way

$$
r=\varepsilon^{3 / 2}, \quad \delta=\varepsilon^{4 / 3} .
$$

This corresponds to the Case $(i i)$ with $\eta_{0}=1.5, \kappa_{0}=\kappa_{1}=1$. A comparison between the sequence of the solutions $u_{\varepsilon}$ and $u$ is done for jumps in displacement and stress. Components of the jumps are computed for different $\varepsilon$, and it is shown that the following norms tend to 0 as $\varepsilon$ tends to 0 :

$$
\left\|\left[u_{\varepsilon, 1}\right]-\left[u_{1}\right]\right\|_{L^{2}(\Sigma)}, \quad\left\|\left[u_{\varepsilon, 2}\right]\right\|_{L^{2}(\Sigma)}, \quad\left\|\sigma_{\varepsilon, 12}^{+}-\sigma_{12}^{+}\right\|_{L^{2}(\Sigma)}, \quad\left\|\sigma_{\varepsilon, 22}^{+}-\sigma_{22}^{+}\right\|_{L^{2}(\Sigma)} .
$$

The stiffness coefficients and the applied force are chosen as follows

$$
E=2 \cdot 10^{11}, \quad \nu=0.3, \quad f_{\varepsilon}=\left(10^{3}, 10^{3}\right) .
$$

Fig. 2 (a) provides the solution of the equation (5.5)-(5.7) in macroscopic blocks, and jumps in the equivalent von Mises stresses across the interface can be observed. Fig. 2 (b) shows the local $\varepsilon$-solution in the layer for $\varepsilon=0.004$.

A comparison of the results for different values of $\varepsilon$ are gathered in the Table 1 in terms of the closing error between the simulated result and the limit problem. Simulations for smaller $\varepsilon$ are already computationally expensive and require additional computational capacities. For example, for $\varepsilon=0.0008$ it was not possible to perform computations due to lack of memory on the system used.

Acknowledgements. This work was supported by the Deutsche Forschungsgemeinschaft (Grants No. OR 190/4-2 and OR 190/6-1). 


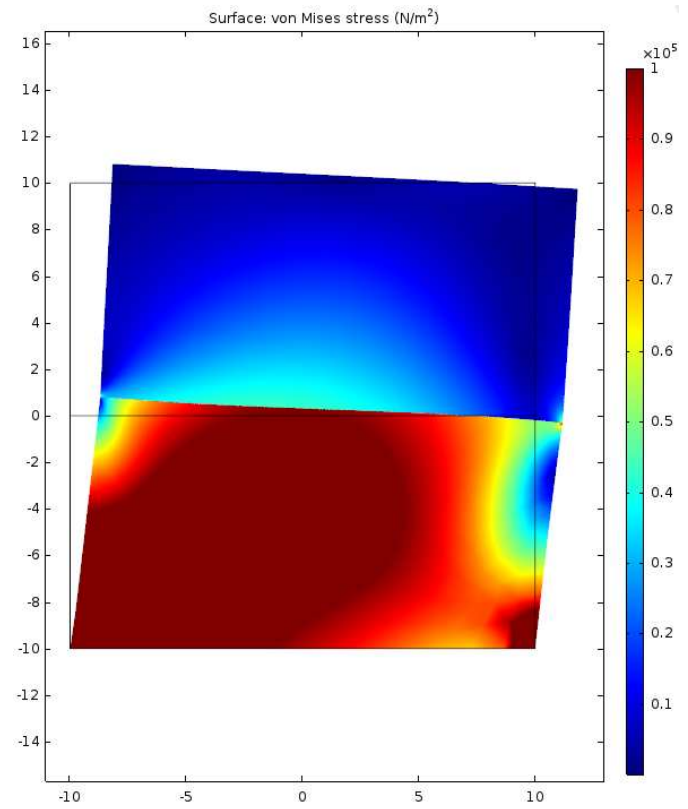

(a) Stresses $\sigma_{e q}^{+}, \sigma_{e q}^{-}$in the regions $\Omega^{+} \cup \Omega^{-}$

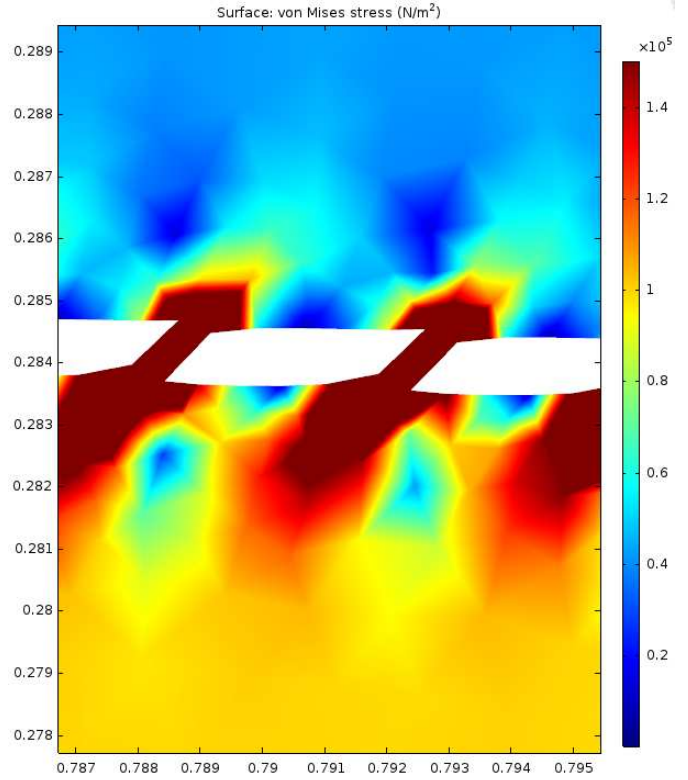

(b) Stresses $\sigma_{\varepsilon, e q}$ in the layer $\Omega_{\varepsilon}^{i}$

Figure 2: Simulation results

\begin{tabular}{|c|c|c|c|c|}
\hline$\varepsilon$ & $\left\|\left[u_{\varepsilon, 1}\right]-\left[u_{1}\right]\right\|$ & $\left\|\left[u_{\varepsilon, 2}\right]\right\|$ & $\left\|\sigma_{\varepsilon, 12}^{+}-\sigma_{12}^{+}\right\|$ & $\left\|\sigma_{\varepsilon, 22}^{+}-\sigma_{22}^{+}\right\|$ \\
\hline 0.1 & $2.9 \cdot 10^{-5}$ & $3 \cdot 10^{-5}$ & 1.05 & 1.1 \\
\hline 0.02 & $2.5 \cdot 10^{-5}$ & $1.6 \cdot 10^{-5}$ & 0.59 & 0.6 \\
\hline 0.004 & $7.6 \cdot 10^{-6}$ & $7.7 \cdot 10^{-6}$ & 0.3 & 0.1 \\
\hline
\end{tabular}

Table 1: Norms of the residual

\section{A Appendix: Proofs of result of Sec. 3}

Proof of Lemma 3.1. Applying the 2D-Poincaré-Wirtinger's inequality we obtain the following estimate:

$$
\|u-\mathcal{U}\|_{L^{2}\left(B_{r, \varepsilon}^{\prime}\right)} \leq C r\|\nabla u\|_{L^{2}\left(B_{r, \varepsilon}^{\prime}\right)},
$$

where the constant does not depend on $r$ and $\varepsilon$.

Step 1. Estimate of $\mathcal{R}(0)$.

Recalling the definition of $\mathcal{R}$ from (3.2) and using $\int_{D_{r}} x_{1} d x_{1} d x_{2}=\int_{D_{r}} x_{2} d x_{1} d x_{2}=0$, we can write

$$
\forall x_{3} \in[-\varepsilon, 0], \quad \mathcal{R}_{1}\left(x_{3}\right)=\frac{1}{I_{2} r^{4}} \int_{D_{r}} x_{2}\left(u_{3}(x)-\mathcal{U}_{3}\left(x_{3}\right)\right) d x_{1} d x_{2} .
$$

By Cauchy's inequality

$$
\begin{aligned}
\forall x_{3} \in[-\varepsilon, 0], \quad\left|\mathcal{R}_{1}\left(x_{3}\right)\right|^{2} & \leq \frac{1}{I_{2}^{2} r^{8}} \int_{D_{r}} x^{2} d x_{1} d x_{2} \times \int_{D_{r}}\left(u_{3}(x)-\mathcal{U}_{3}\left(x_{3}\right)\right)^{2} d x_{1} d x_{2} \\
& \leq \frac{C}{r^{4}} \int_{D_{r}}\left(u_{3}(x)-\mathcal{U}_{3}\left(x_{3}\right)\right)^{2} d x_{1} d x_{2} .
\end{aligned}
$$

Integrating with respect to $x_{3}$ gives

$$
\int_{-\varepsilon}^{0}\left|\mathcal{R}_{1}\left(x_{3}\right)\right|^{2} d x_{3} \leq \frac{C}{r^{4}} \int_{B_{r, \varepsilon}}\left(u(x)-\mathcal{U}\left(x_{3}\right)\right)^{2} d x .
$$

Using (A.1), we can write

$$
\left\|\mathcal{R}_{1}\right\|_{L^{2}(-\varepsilon, 0)} \leq \frac{C}{r}\|\nabla u\|_{L^{2}\left(B_{r, \varepsilon}^{\prime}\right)} .
$$

The derivative of $\mathcal{R}_{1}$ is equal to

$$
\frac{d \mathcal{R}_{1}}{d x_{3}}\left(x_{3}\right)=\frac{1}{I_{2} r^{4}} \int_{D_{r}} x_{2} \frac{\partial u_{3}(x)}{\partial x_{3}} d x_{1} d x_{2}
$$


for a.e. $x_{3} \in(-\varepsilon, 0)$. Then proceeding as above, we obtain for a.e. $x_{3} \in(-\varepsilon, 0)$

$$
\left|\frac{d \mathcal{R}_{1}}{d x_{3}}\left(x_{3}\right)\right|^{2} \leq \frac{C}{r^{4}} \int_{D_{r}}\left|\frac{\partial u_{3}(x)}{\partial x_{3}}\right|^{2} d x_{1} d x_{2} .
$$

Hence

$$
\left\|\frac{d \mathcal{R}_{1}}{d x_{3}}\right\|_{L^{2}(-\varepsilon, 0)} \leq \frac{C}{r^{2}}\left\|\frac{\partial u_{3}}{\partial x_{3}}\right\|_{L^{2}\left(B_{r, \varepsilon}^{\prime}\right)} \leq \frac{C}{r^{2}}\|\nabla u\|_{L^{2}\left(B_{r, \varepsilon}^{\prime}\right)} .
$$

We recall following classical estimates for $\phi \in H^{1}(-a, 0)$, where $a>0$

$$
\begin{aligned}
& |\phi(0)|^{2} \leq \frac{2}{a}\|\phi\|_{L^{2}(-a, 0)}^{2}+\frac{a}{2}\left\|\phi^{\prime}\right\|_{L^{2}(-a, 0)}^{2}, \\
& \|\phi\|_{L^{2}(-a, 0)}^{2} \leq 2 a|\phi(0)|^{2}+a^{2}\left\|\phi^{\prime}\right\|_{L^{2}(-a, 0)}^{2} .
\end{aligned}
$$

Due to (A.2)-(A.3), (A.4) 1 with $a=r$ and $\varepsilon>r, \mathcal{R}_{1}(0)$ satisfies

$$
\left|\mathcal{R}_{1}(0)\right|^{2} \leq \frac{C}{r^{3}}\|\nabla u\|_{L^{2}\left(B_{r, \varepsilon}^{\prime}\right)}^{2} .
$$

The estimates for $\mathcal{R}_{2}(0), \mathcal{R}_{3}(0)$ are obtained in the same way. Hence we get $(3.6)_{1}$.

Step 2. Estimate of $\|\mathcal{R}\|_{L^{2}(0, \delta)}$.

Poincaré's inequality yields

$$
\|\mathcal{R}-\mathcal{R}(0)\|_{L^{2}(0, \delta)} \leq \delta\left\|\frac{d \mathcal{R}}{d x_{3}}\right\|_{L^{2}(0, \delta)} .
$$

From $(3.5)_{3},(\text { A.4 })_{2}$ and $(3.6)_{1}$ we get

$$
\|\mathcal{R}\|_{L^{2}(0, \delta)}^{2} \leq 2 \delta|\mathcal{R}(0)|^{2}+\delta^{2}\left\|\frac{d \mathcal{R}}{d x_{3}}\right\|_{L^{2}(0, \delta)}^{2} \leq C \frac{\delta}{r^{3}}\|\nabla u\|_{L^{2}\left(B_{r, \varepsilon}^{\prime}\right)}^{2}+C \frac{\delta^{2}}{r^{4}}\|e(u)\|_{L^{2}\left(B_{r, \delta}\right)}^{2} .
$$

Hence $(3.6)_{2}$ is proved.

Step 3. Estimate of $\mathcal{U}-\mathcal{U}(0)$.

Applying the inequality (3.5) from Theorem 3.1 the following estimates on $\mathcal{U}$ hold:

$$
\begin{aligned}
\left\|\frac{d \mathcal{U}_{3}}{d x_{3}}\right\|_{L^{2}(0, \delta)} & \leq \frac{C}{r}\|e(u)\|_{L^{2}\left(B_{r, \delta}\right)}, \\
\left\|\frac{d \mathcal{U}_{\alpha}}{d x_{3}}\right\|_{L^{2}(0, \delta)} & \leq\|\mathcal{R}\|_{L^{2}(0, \delta)}+\frac{C}{r}\|e(u)\|_{L^{2}\left(B_{r, \delta}\right)} .
\end{aligned}
$$

Combining (A.6) $)_{2}$ with (A.5) gives

$$
\left\|\frac{d \mathcal{U}_{\alpha}}{d x_{3}}\right\|_{L^{2}(0, \delta)}^{2} \leq C \frac{\delta}{r^{3}}\|\nabla u\|_{L^{2}\left(B_{r, \delta}^{\prime}\right)}^{2}+C \frac{\delta^{2}}{r^{4}}\|e(u)\|_{L^{2}\left(B_{r, \delta}\right)}^{2}+\frac{C}{r^{2}}\|e(u)\|_{L^{2}\left(B_{r, \delta}\right)}^{2} .
$$

Taking into account assumption $(2.7)_{2}$, we obtain $(3.6)_{3}$. Then by $(3.6)_{3},(\text { A.6 })_{1}$ and Poincaré's inequality formulas $(3.6)_{4},(3.6)_{5}$ follow.

Step 4. We prove the estimate $(3.6)_{6}$.

By Korn's inequality, there exists a rigid displacement $\mathbf{r}$

$$
\begin{aligned}
& \mathbf{r}(x)=\mathbf{a}+\mathbf{b} \wedge\left(x+\frac{\varepsilon}{2} \mathbf{e}_{\mathbf{3}}\right), \\
& \mathbf{a}=\frac{1}{\varepsilon^{3}} \int_{W_{\varepsilon}} u(x) d x, \\
& \mathbf{b}=\frac{6}{\varepsilon^{5}} \int_{W_{\varepsilon}}\left(x+\frac{\varepsilon}{2} \mathbf{e}_{3}\right) \wedge u(x) d x,
\end{aligned}
$$

such that

$$
\begin{array}{r}
\|u-\mathbf{r}\|_{L^{2}\left(W_{\varepsilon}\right)} \leq C \varepsilon\|e(u)\|_{L^{2}\left(W_{\varepsilon}\right)}, \\
\|\nabla(u-\mathbf{r})\|_{L^{2}\left(W_{\varepsilon}\right)} \leq C\|e(u)\|_{L^{2}\left(W_{\varepsilon}\right)} .
\end{array}
$$


Besides, by Poincaré-Wirtinger inequality we have

$$
\|u-\mathbf{a}\|_{L^{2}\left(W_{\varepsilon}\right)} \leq C \varepsilon\|\nabla u\|_{L^{2}\left(W_{\varepsilon}\right)} .
$$

Using the following Sobolev embedding theorem

$$
W^{s, p} \subset W^{t, q}, \quad \text { for } s, p, t, q: s \in \mathbb{R}, s>t, p \in \mathbb{N} \text { and } \frac{1}{q}=\frac{1}{p}-\frac{s-t}{n}
$$

we get

$$
H^{1 / 2} \subset L^{4} \quad \text { or } \quad\|\varphi\|_{L^{4}(Y)} \leq C\|\varphi\|_{H^{1 / 2}(Y)}, \quad \forall \varphi \in H^{1 / 2}(Y) .
$$

Moreover, by definition of the $H^{1 / 2}$ norm for $W=Y \times(-1,0)$, we have

$$
\|\varphi\|_{H^{1 / 2}(Y)} \leq C\left(\|\varphi\|_{L^{2}(W)}+\|\nabla \varphi\|_{L^{2}(W)}\right), \quad \forall \varphi \in H^{1}(W) .
$$

Therefore,

$$
\|\varphi\|_{L^{4}(Y)} \leq C\left(\|\varphi\|_{L^{2}(W)}+\|\nabla \varphi\|_{L^{2}(W)}\right), \quad \forall \varphi \in H^{1}(W) .
$$

With the change of variables

$$
y_{\varepsilon}=\varepsilon y, \quad \text { for }\left(y_{1}, y_{2}\right) \in Y, \quad\left(y_{1}, y_{2}, y_{3}\right) \in W,
$$

and defining

we obtain

$$
\varphi_{\varepsilon}\left(y_{\varepsilon}\right)=\varphi\left(\frac{y_{\varepsilon}}{\varepsilon}\right)
$$

$$
\frac{1}{\sqrt{\varepsilon}}\left\|\varphi_{\varepsilon}\right\|_{L^{4}\left(Y_{\varepsilon}\right)} \leq C\left(\frac{1}{\varepsilon^{3 / 2}}\left\|\varphi_{\varepsilon}\right\|_{L^{2}\left(W_{\varepsilon}\right)}+\frac{1}{\sqrt{\varepsilon}}\left\|\nabla \varphi_{\varepsilon}\right\|_{L^{2}\left(W_{\varepsilon}\right)}\right), \quad \forall \varphi_{\varepsilon} \in H^{1}\left(W_{\varepsilon}\right)
$$

or

$$
\|\varphi\|_{L^{4}\left(Y_{\varepsilon}\right)} \leq C\left(\frac{1}{\varepsilon}\|\varphi\|_{L^{2}\left(W_{\varepsilon}\right)}+\|\nabla \varphi\|_{L^{2}\left(W_{\varepsilon}\right)}\right), \quad \forall \varphi \in H^{1}\left(W_{\varepsilon}\right) .
$$

Therefore, (A.7) and the inequality above lead to

$$
\|u-\mathbf{r}\|_{L^{4}\left(Y_{\varepsilon}\right)} \leq C\|e(u)\|_{L^{2}\left(W_{\varepsilon}\right)} .
$$

From the identity

$$
\frac{1}{\pi r^{2}} \int_{D_{r}}\left(u\left(x^{\prime}, 0\right)-\mathbf{r}\left(x^{\prime}, 0\right)\right) d x^{\prime}=\mathcal{U}(0)-\mathbf{a}-\mathbf{b} \wedge \frac{\varepsilon}{2} e_{3},
$$

the estimate (A.9) and Hölder's inequality we get

$$
\left|\mathcal{U}(0)-\mathbf{a}-\mathbf{b} \wedge \frac{\varepsilon}{2} e_{3}\right| \leq \frac{1}{\pi r^{2}}\left(\int_{D_{r}} 1^{4 / 3} d x^{\prime}\right)^{3 / 4}\left(\int_{D_{r}}\left|u\left(x^{\prime}, 0\right)-\mathbf{r}\left(x^{\prime}, 0\right)\right|^{4} d x^{\prime}\right)^{1 / 4} \leq \frac{C}{r^{1 / 2}}\|e(u)\|_{L^{2}\left(W_{\varepsilon}\right)} .
$$

From Cauchy-Schwarz's inequality and taking into account (A.8), we derive

$$
\begin{aligned}
|\mathbf{b}| \leq \frac{C}{\varepsilon^{5}}\left(\int_{W_{\varepsilon}}\left|x+\frac{\varepsilon}{2} \mathbf{e}_{3}\right|^{2} d x\right)^{1 / 2} & \left(\int_{W_{\varepsilon}}|u(x)-\mathbf{a}|^{2} d x\right)^{1 / 2} \\
\leq & \frac{C}{\varepsilon^{5}} \cdot \varepsilon \cdot \varepsilon^{3 / 2}\|u-\mathbf{a}\|_{L^{2}\left(W_{\varepsilon}\right)} \leq \frac{C}{\varepsilon^{5 / 2}} \varepsilon\|\nabla u\|_{L^{2}\left(W_{\varepsilon}\right)} \leq \frac{C}{\varepsilon^{3 / 2}}\|\nabla u\|_{L^{2}\left(W_{\varepsilon}\right)} .
\end{aligned}
$$

Using (A.10) and (A.11), we obtain

$$
|\mathcal{U}(0)-\mathbf{a}| \leq\left|\mathcal{U}(0)-\mathbf{a}-\mathbf{b} \wedge \frac{\varepsilon}{2} \mathbf{e}_{3}\right|+\left|\mathbf{b} \wedge \frac{\varepsilon}{2} \mathbf{e}_{3}\right| \leq \frac{C}{r^{1 / 2}}\|e(u)\|_{L^{2}\left(W_{\varepsilon}\right)}+\frac{C}{\varepsilon^{1 / 2}}\|\nabla u\|_{L^{2}\left(W_{\varepsilon}\right)} .
$$

The estimates (A.4) and (A.8) yield

$$
\|u(\cdot, \cdot, 0)-\mathbf{a}\|_{L^{2}\left(Y_{\varepsilon}\right)}^{2} \leq C \varepsilon\|\nabla u\|_{L^{2}\left(W_{\varepsilon}\right)}^{2} .
$$

Combining (A.12) and (A.13) gives

$$
\begin{aligned}
\|u(\cdot, \cdot, 0)-\mathcal{U}(0)\|_{L^{2}\left(Y_{\varepsilon}\right)}^{2} & \leq C\left(\|u(\cdot, \cdot, 0)-\mathbf{a}\|_{L^{2}\left(Y_{\varepsilon}\right)}^{2}+\|\mathcal{U}(0)-\mathbf{a}\|_{L^{2}\left(Y_{\varepsilon}\right)}^{2}\right) \\
& \leq C \varepsilon\|\nabla u\|_{L^{2}\left(W_{\varepsilon}\right)}^{2}+C \frac{\varepsilon^{2}}{r}\|e(u)\|_{L^{2}\left(W_{\varepsilon}\right)}^{2}+C \varepsilon\|\nabla u\|_{L^{2}\left(W_{\varepsilon}\right)}^{2} \\
& \leq C \varepsilon\|\nabla u\|_{L^{2}\left(W_{\varepsilon}\right)}^{2}+C \frac{\varepsilon^{2}}{r}\|e(u)\|_{L^{2}\left(W_{\varepsilon}\right)}^{2} .
\end{aligned}
$$

Hence we get $(3.6)_{6}$. 
Proof of Lemma 3.3. Using $(3.6)_{6}$ and then summing over all of the periodicity cells gives

$$
\|u(\cdot, \cdot, 0)-\tilde{\mathcal{U}}(\cdot, \cdot, 0)\|_{L^{2}\left(\widehat{\omega}_{\varepsilon}\right)}^{2} \leq C \varepsilon\|\nabla u\|_{L^{2}\left(\Omega^{-}\right)}^{2}+C \frac{\varepsilon^{2}}{r}\|u\|_{V}^{2} .
$$

In the same way, the following estimate is derived:

$$
\|u(\cdot, \cdot, \delta)-\widetilde{\mathcal{U}}(\cdot, \cdot, \delta)\|_{L^{2}\left(\widehat{\omega}_{\varepsilon}\right)}^{2} \leq C \varepsilon\|\nabla u\|_{L^{2}\left(\Omega_{\delta}^{+}\right)}^{2}+C \frac{\varepsilon^{2}}{r}\|u\|_{V}^{2} .
$$

Applying (3.7) 2 we can write

$$
\left\|\widetilde{\mathcal{U}}_{3}(\cdot, \cdot, \delta)-\widetilde{\mathcal{U}}_{3}(\cdot, \cdot, 0)\right\|_{L^{2}\left(\widehat{\omega}_{\varepsilon}\right)}^{2} \leq \delta\left\|\frac{\partial \widetilde{\mathcal{U}}_{3}}{\partial x_{3}}\right\|_{L^{2}\left(\widehat{\omega}_{\varepsilon} \times(0, \delta)\right)}^{2} \leq C \frac{\varepsilon^{2} \delta}{r^{2}}\|u\|_{V}^{2} .
$$

From $(3.7)_{6}$ we have

$$
\left\|\tilde{\mathcal{U}}_{\alpha}(\cdot, \cdot, \delta)-\widetilde{\mathcal{U}}_{\alpha}(\cdot, \cdot, 0)\right\|_{L^{2}\left(\widehat{\omega}_{\varepsilon}\right)}^{2} \leq C \frac{\varepsilon^{2} \delta^{2}}{r^{3}}\|u\|_{V}^{2}+C \frac{\varepsilon^{2} \delta^{3}}{r^{4}}\|u\|_{V}^{2} \leq C \frac{\varepsilon^{2} \delta^{3}}{r^{4}}\|u\|_{V}^{2} .
$$

Using (A.16) and the estimates above we obtain (3.11), (3.12).

Proof of Lemma 3.4. From Korn's inequality and the trace theorem we derive

$$
\begin{aligned}
& \|u\|_{L^{2}(\Sigma)} \leq C\|u\|_{H^{1}\left(\Omega^{-}\right)} \leq C_{1}\|e(u)\|_{L^{2}\left(\Omega^{-}\right)}, \\
& \|u\|_{H^{1}\left(\Omega_{\delta}^{+}\right)} \leq C\left(\|e(u)\|_{L^{2}\left(\Omega_{\delta}^{+}\right)}+\|u\|_{L^{2}\left(\Sigma_{\delta}^{+}\right)}\right) .
\end{aligned}
$$

We know that there exists a rigid displacement $\mathbf{r}$

$$
\forall x \in \mathbb{R}^{3}, \quad \mathbf{r}(x)=\mathbf{a}+\mathbf{b} \wedge\left(x-\delta \mathbf{e}_{\mathbf{3}}\right), \quad \mathbf{a}, \mathbf{b} \in \mathbb{R}^{3},
$$

such that

$$
\|u-\mathbf{r}\|_{L^{2}\left(\Sigma_{\delta}^{+}\right)} \leq C\|u-\mathbf{r}\|_{H^{1}\left(\Omega_{\delta}^{+}\right)} \leq C\|e(u)\|_{L^{2}\left(\Omega_{\delta}^{+}\right)},
$$

where the constant does not depend on $\delta$ (since $\left|\Sigma_{\delta}^{+}\right|$is independent of $\delta$ and $\Omega_{\delta}^{+} \subset \Omega^{+}$and $\left|\Omega^{+} \backslash \Omega_{\delta}^{+}\right|=|\omega| \delta$ ). Then, we get

$$
\|(u-\mathbf{r})(\cdot, \cdot, \delta)\|_{L^{2}\left(\widehat{\omega}_{\varepsilon}\right)} \leq\|u-\mathbf{r}\|_{L^{2}\left(\Sigma_{\delta}^{+}\right)} \leq C\|e(u)\|_{L^{2}\left(\Omega_{\delta}^{+}\right)} .
$$

Using

$$
\|u(\cdot, \cdot, 0)\|_{L^{2}\left(\widehat{\omega}_{\varepsilon}\right)} \leq\|u\|_{L^{2}(\Sigma)},
$$

from (3.11) and (3.12) we obtain

$$
\begin{gathered}
\left\|u_{\alpha}(\cdot, \cdot, \delta)\right\|_{L^{2}\left(\widehat{\omega}_{\varepsilon}\right)} \leq C \varepsilon^{1 / 2}\|\nabla u\|_{L^{2}\left(\Omega_{\delta}^{+}\right)}+C \frac{\varepsilon \delta^{3 / 2}}{r^{2}}\|u\|_{V}+C\|u\|_{V}, \\
\left\|u_{3}(\cdot, \cdot, \delta)\right\|_{L^{2}\left(\widehat{\omega}_{\varepsilon}\right)} \leq C \varepsilon^{1 / 2}\|\nabla u\|_{L^{2}\left(\Omega_{\delta}^{+}\right)}+C \frac{\varepsilon^{3 / 2} \delta^{1 / 2}}{r}\|u\|_{V}+C\|u\|_{V} .
\end{gathered}
$$

Combining this with (A.19) gives

$$
\begin{gathered}
\left\|\mathbf{r}_{\alpha}(\cdot, \cdot, \delta)\right\|_{L^{2}\left(\widehat{\omega}_{\varepsilon}\right)} \leq C \varepsilon^{1 / 2}\|\nabla u\|_{L^{2}\left(\Omega_{\delta}^{+}\right)}+C \frac{\varepsilon \delta^{3 / 2}}{r^{2}}\|u\|_{V}+C\|u\|_{V}, \\
\left\|\mathbf{r}_{3}(\cdot, \cdot, \delta)\right\|_{L^{2}\left(\widehat{\omega}_{\varepsilon}\right)} \leq C \varepsilon^{1 / 2}\|\nabla u\|_{L^{2}\left(\Omega_{\delta}^{+}\right)}+C \frac{\varepsilon \delta^{1 / 2}}{r}\|u\|_{V}+C\|u\|_{V} .
\end{gathered}
$$

Therefore,

$$
\begin{aligned}
& \left|\mathbf{a}_{\mathbf{1}}\right|+\left|\mathbf{a}_{\mathbf{2}}\right|+\left|\mathbf{b}_{\mathbf{3}}\right| \leq C \varepsilon^{1 / 2}\|\nabla u\|_{L^{2}\left(\Omega_{\delta}^{+}\right)}+C \frac{\varepsilon \delta^{3 / 2}}{r^{2}}\|u\|_{V}+C\|u\|_{V}, \\
& \left|\mathbf{a}_{\mathbf{3}}\right|+\left|\mathbf{b}_{\mathbf{1}}\right|+\left|\mathbf{b}_{\mathbf{2}}\right| \leq C \varepsilon^{1 / 2}\|\nabla u\|_{L^{2}\left(\Omega_{\delta}^{+}\right)}+C \frac{\varepsilon \delta^{1 / 2}}{r}\|u\|_{V}+C\|u\|_{V} .
\end{aligned}
$$

These estimates together with (A.18) allow us to obtain estimates on $u_{1}, u_{2}, u_{3}$. This yields

$$
\begin{aligned}
& \left\|u_{\alpha}\right\|_{H^{1}\left(\Omega_{\delta}^{+}\right)} \leq C \varepsilon^{1 / 2}\|\nabla u\|_{L^{2}\left(\Omega_{\delta}^{+}\right)}+C \frac{\varepsilon \delta^{3 / 2}}{r^{2}}\|u\|_{V}+C\|u\|_{V}, \\
& \left\|u_{3}\right\|_{H^{1}\left(\Omega_{\delta}^{+}\right)} \leq C \varepsilon^{1 / 2}\|\nabla u\|_{L^{2}\left(\Omega_{\delta}^{+}\right)}+C \frac{\varepsilon \delta^{1 / 2}}{r}\|u\|_{V}+C\|u\|_{V} .
\end{aligned}
$$


Therefore,

$$
\|\nabla u\|_{L^{2}\left(\Omega_{\delta}^{+}\right)} \leq C \varepsilon^{1 / 2}\|\nabla u\|_{L^{2}\left(\Omega_{\delta}^{+}\right)}+C \frac{\varepsilon \delta^{3 / 2}}{r^{2}}\|u\|_{V}+C\|u\|_{V} .
$$

For $\varepsilon$ small enough the following holds true:

$$
\|\nabla u\|_{L^{2}\left(\Omega_{\delta}^{+}\right)} \leq C \frac{\varepsilon \delta^{3 / 2}}{r^{2}}\|u\|_{V}+C\|u\|_{V} .
$$

Inserting this in (A.23) we derive (3.13)-(3.14).

Proof of Lemma 3.5. From the estimates in Theorem 3.1, $(3.6)_{2}$ and $(3.6)_{3}$ and after summation over all the beams, we get (making use of the assumption $(2.7)_{2}$ )

$$
\|\nabla u\|_{L^{2}\left(\Omega_{r, \varepsilon, \delta}^{i}\right)}^{2} \leq C\left(\frac{\delta}{r}\|\nabla u\|_{L^{2}\left(\Omega^{-}\right)}^{2}+\frac{\delta^{2}}{r^{2}}\|e(u)\|_{L^{2}\left(\Omega_{r, \varepsilon, \delta}^{i}\right)}^{2}\right) \leq C \frac{\delta^{2}}{r^{2}}\|u\|_{V}^{2}
$$

From (A.14) and (A.17) 1 , it follows that

$$
\begin{gathered}
\sum_{\xi \in \Xi_{\varepsilon}} \varepsilon^{2}\left|\mathcal{U}_{\xi}(0)\right|^{2}=\|\widetilde{\mathcal{U}}(\cdot, \cdot, 0)\|_{L^{2}\left(\widehat{\omega}_{\varepsilon}\right)}^{2} \leq C \frac{\varepsilon^{2}}{r}\|u\|_{V}^{2}+C\|u\|_{V}^{2}, \\
\sum_{\xi \in \Xi_{\varepsilon}}\left|\mathcal{U}_{\xi}(0)\right|^{2} \leq C\left(\frac{1}{r}+\frac{1}{\varepsilon^{2}}\right)\|u\|_{V}^{2} .
\end{gathered}
$$

Using $(3.5)_{4},(3.6)_{3},($ A.4), we obtain

$$
\begin{aligned}
& \sum_{\xi \in \Xi_{\varepsilon}}\left\|\mathcal{U}_{\xi, 3}\right\|_{L^{2}(0, \delta)}^{2} \leq C\left(\frac{\delta}{\varepsilon^{2}}+\frac{\delta^{2}}{r^{2}}\right)\|u\|_{V}^{2}, \\
& \sum_{\xi \in \Xi_{\varepsilon}}\left\|\mathcal{U}_{\xi, \alpha}\right\|_{L^{2}(0, \delta)}^{2} \leq C\left(\frac{\delta}{\varepsilon^{2}}+\frac{\delta^{4}}{r^{4}}\right)\|u\|_{V}^{2} .
\end{aligned}
$$

Additionally,

$$
\sum_{\xi \in \Xi_{\varepsilon}}\left\|\bar{u}_{\xi}\right\|_{L^{2}\left(B_{r, \delta}\right)}^{2} \leq C r^{2}\|e(u)\|_{L^{2}\left(\Omega_{r, \varepsilon, \delta}^{i}\right)}^{2} \leq C r^{2}\|u\|_{V}^{2}
$$

Then $(3.6)_{2},(\mathrm{~A} .25)$ and (A.26) give

$$
\begin{gathered}
\sum_{\xi \in \Xi_{\varepsilon}}\left\|u_{\xi, \alpha}\right\|_{L^{2}\left(B_{r, \delta}\right)}^{2} \leq C\left(\frac{r^{2} \delta}{\varepsilon^{2}}+\frac{\delta^{4}}{r^{2}}+\delta^{2}+r^{2}\right)\|u\|_{V}^{2} \leq C \frac{r^{2} \delta}{\varepsilon^{2}}\left(1+\frac{\varepsilon^{2} \delta^{3}}{r^{4}}\right)\|u\|_{V}^{2}, \\
\sum_{\xi \in \Xi_{\varepsilon}}\left\|u_{\xi, 3}\right\|_{L^{2}\left(B_{r, \delta}\right)}^{2} \leq C\left(\frac{r^{2} \delta}{\varepsilon^{2}}+\delta^{2}+r^{2}\right)\|u\|_{V}^{2} \leq C \frac{r^{2} \delta}{\varepsilon^{2}}\left(1+\frac{\varepsilon^{2} \delta}{r^{2}}\right)\|u\|_{V}^{2} .
\end{gathered}
$$

From the last inequalities, we derive $(3.17)_{2}$ and $(3.17)_{3}$.

\section{B Properties of the periodic unfolding operators. Estimates for the compactness.}

Lemma B.1. (Properties of the operators $\mathcal{T}_{\varepsilon}, \mathcal{T}_{\varepsilon}^{\prime}$ )

1. $\forall v, w \in L^{2}(\omega \times(0, \delta))$

$$
\mathcal{T}_{\varepsilon}(v w)=\mathcal{T}_{\varepsilon}(v) \mathcal{T}_{\varepsilon}(w)
$$

$\forall v, w \in L^{2}\left(\omega \times B_{r, \delta}\right)$

$$
\mathcal{T}_{\varepsilon}^{\prime}(v w)=\mathcal{T}_{\varepsilon}^{\prime}(v) \mathcal{T}_{\varepsilon}^{\prime}(w)
$$

2. $\forall u \in L^{1}(\omega \times(0, \delta))$

$$
\delta \int_{\omega \times(0,1)} \mathcal{T}_{\varepsilon}(u) d s_{1} d s_{2} d X_{3}=\int_{\widehat{\omega}_{\varepsilon} \times(0, \delta)} u d s_{1} d s_{2} d x_{3}
$$

$\forall u \in L^{1}\left(\omega \times B_{r, \delta}\right)$

$$
r^{2} \delta \int_{\omega \times B_{1}} \mathcal{T}_{\varepsilon}^{\prime}(u) d s_{1} d s_{2} d X_{1} d X_{2} d X_{3}=\int_{\widehat{\omega}_{\varepsilon} \times B_{r, \delta}} u d s_{1} d s_{2} d x_{1} d x_{2} d x_{3} .
$$


3. $\forall u \in L^{2}(\omega \times(0, \delta))$

$$
\left\|\mathcal{T}_{\varepsilon}(u)\right\|_{L^{2}(\omega \times(0,1))} \leq \frac{1}{\sqrt{\delta}}\|u\|_{L^{2}(\omega \times(0, \delta))},
$$

$\forall u \in L^{2}\left(\omega \times B_{r, \delta}\right)$

$$
\left\|\mathcal{T}_{\varepsilon}^{\prime}(u)\right\|_{L^{2}\left(\omega \times B_{1}\right)} \leq \frac{1}{r \sqrt{\delta}}\|u\|_{L^{2}\left(\omega \times B_{r, \delta}\right)} .
$$

4. Let $u$ be in $L^{2}\left(\omega, H^{1}(0, \delta)\right)$. Then we have

$$
\delta \mathcal{T}_{\varepsilon}\left(\nabla_{x_{3}} u\right)=\nabla_{X_{3}} \mathcal{T}_{\varepsilon}(u) .
$$

Let $u$ be in $L^{2}\left(\omega, H^{1}\left(B_{r, \delta}\right)\right)$. Then we have

$$
r \mathcal{T}_{\varepsilon}^{\prime}\left(\nabla_{x_{\alpha}} u\right)=\nabla_{X_{\alpha}} \mathcal{T}_{\varepsilon}^{\prime}(u), \quad \delta \mathcal{T}_{\varepsilon}^{\prime}\left(\nabla_{x_{3}} u\right)=\nabla_{X_{3}} \mathcal{T}_{\varepsilon}^{\prime}(u), \text { where } \alpha=1,2
$$

Proof. Properties 1-3 are obtained similarly to the proof of Lemma 5.1 of [2].

Property 4 is the direct consequence of the chain rule:

$$
\begin{gathered}
\frac{\partial\left(\mathcal{T}_{\varepsilon}^{\prime}(u)\right)}{\partial X_{\alpha}}=r \mathcal{T}_{\varepsilon}^{\prime}\left(\frac{\partial u}{\partial x_{\alpha}}\right), \quad \alpha=1,2, \\
\frac{\partial\left(\mathcal{T}_{\varepsilon}(u)\right)}{\partial X_{3}}=\delta \mathcal{T}_{\varepsilon}\left(\frac{\partial u}{\partial x_{3}}\right), \quad \frac{\partial\left(\mathcal{T}_{\varepsilon}^{\prime}(u)\right)}{\partial X_{3}}=\delta \mathcal{T}_{\varepsilon}^{\prime}\left(\frac{\partial u}{\partial x_{3}}\right) .
\end{gathered}
$$

From Lemmas 3.2 and B.1 we obtain the following result.

Lemma B.2. There exists a constant $C$, independent of $\varepsilon, \delta$ and $r$, such that

$$
\begin{gathered}
\left\|\mathcal{T}_{\varepsilon}\left(\widetilde{\mathcal{U}}_{\varepsilon}\right)\right\|_{L^{2}\left(\omega, H^{1}(0,1)\right)} \leq C, \\
\left\|\mathcal{T}_{\varepsilon}\left(\widetilde{\mathcal{U}}_{\varepsilon, 3}\right)-\widetilde{\mathcal{U}}_{\varepsilon, 3}(\cdot, \cdot, 0)\right\|_{L^{2}\left(\omega, H^{1}(0,1)\right)} \leq C \frac{r}{\delta}, \\
\left\|\mathcal{T}_{\varepsilon}\left(\widetilde{\mathcal{R}}_{\varepsilon}\right)\right\|_{L^{2}\left(\omega, H^{1}(0,1)\right)} \leq \frac{C}{\delta}, \\
\left\|\frac{\partial \mathcal{T}_{\varepsilon}\left(\widetilde{\mathcal{U}}_{\varepsilon}\right)}{\partial X_{3}}-\delta \mathcal{T}_{\varepsilon}\left(\widetilde{\mathcal{R}}_{\varepsilon}\right) \wedge \mathbf{e}_{3}\right\|_{L^{2}(\omega \times(0,1))} \leq C \frac{r}{\delta}, \\
\left\|\mathcal{T}_{\varepsilon}^{\prime}\left(\widetilde{u}_{\varepsilon}\right)\right\|_{L^{2}\left(\omega \times(0,1), H^{1}\left(D_{1}\right)\right)} \leq C \frac{r^{2}}{\delta^{2}}, \\
\left\|\frac{\partial \mathcal{T}_{\varepsilon}^{\prime}\left(\widetilde{u}_{\varepsilon}\right)}{\partial X_{3}}\right\|_{L^{2}\left(\omega \times B_{1}\right)} \leq C \frac{r}{\delta} .
\end{gathered}
$$

Proof of Proposotion 4.1. The convergences in (4.1)-(4.3), (4.5), (4.6), (4.8), (4.12) and (4.14) follow from estimate (3.23) and those in Lemma B.2.

The equalities in (4.4) are consequences of $(3.8)_{1}-(3.8)_{2}$. To obtain (4.11), take into account that from (4.14) we have

$$
\frac{\partial \widetilde{\mathcal{U}}}{\partial X_{3}}-\widetilde{\mathcal{R}} \wedge \mathbf{e}_{3}=\left(\begin{array}{c}
\frac{\partial \widetilde{\mathcal{U}}_{1}}{\partial X_{3}}-\widetilde{\mathcal{R}}_{2} \\
\frac{\partial \widetilde{\mathcal{U}}_{2}}{\partial X_{3}}+\widetilde{\mathcal{R}}_{1} \\
\frac{\partial \widetilde{\mathcal{U}}_{3}}{\partial X_{3}}
\end{array}\right)=0 .
$$

Then (4.4) yields (4.10). Equalities in (4.7) are the consequences of $\frac{\partial \widetilde{\mathcal{U}}_{3}}{\partial X_{3}}=0$ and estimates (3.9), (3.10). Again due to $(3.9),(3.10)$, we obtain

$$
\widetilde{\mathcal{U}}_{\alpha}\left(x^{\prime}, 0\right)=u_{\alpha \mid \Sigma}^{-}\left(x^{\prime}\right), \quad \widetilde{\mathcal{U}}_{\alpha}\left(x^{\prime}, 1\right)=u_{\alpha \mid \Sigma}^{+}\left(x^{\prime}\right), \quad \text { for a.e. } x^{\prime} \in \omega .
$$

From Lemma B.2 we have $\left\|\mathcal{T}_{\varepsilon}^{\prime}\left(\widetilde{u}_{\varepsilon}\right)\right\|_{L^{2}\left(\omega, H^{1}\left(B_{1}\right)\right)} \leq C \frac{r}{\delta}$. From this and (4.12) we deduce (4.13). 
The strain tensor of the displacement $u_{\varepsilon}$ is

$$
\begin{gathered}
\mathcal{T}_{\varepsilon}^{\prime}\left(\widetilde{e\left(u_{\varepsilon}\right)}\right)_{i j}=\mathcal{T}_{\varepsilon}^{\prime}\left(\widetilde{e\left(u_{\varepsilon}\right)}\right)_{i j}, \quad i, j=1,2, \\
\mathcal{T}_{\varepsilon}^{\prime}\left(\widetilde{\left.e\left(u_{\varepsilon}\right)\right)_{13}=} \frac{1}{2}\left(\left(\frac{1}{\delta} \frac{\partial \mathcal{T}_{\varepsilon}\left(\widetilde{\mathcal{U}}_{\varepsilon, 1}\right)}{\partial X_{3}}-\mathcal{T}_{\varepsilon}\left(\widetilde{\mathcal{R}}_{\varepsilon, 2}\right)\right)-\frac{r}{\delta} \frac{\partial \mathcal{T}_{\varepsilon}\left(\widetilde{\mathcal{R}}_{\varepsilon, 3}\right)}{\partial X_{3}} X_{2}\right)+\mathcal{T}_{\varepsilon}^{\prime}\left(\widetilde{e\left(u_{\varepsilon}\right)}\right)_{13},\right. \\
\mathcal{T}_{\varepsilon}^{\prime}\left(\widetilde{\left.e\left(u_{\varepsilon}\right)\right)_{23}=} \frac{1}{2}\left(\left(\frac{1}{\delta} \frac{\partial \mathcal{T}_{\varepsilon}\left(\widetilde{\mathcal{U}}_{\varepsilon, 2}\right)}{\partial X_{3}}+\mathcal{T}_{\varepsilon}\left(\widetilde{\mathcal{R}}_{\varepsilon, 1}\right)\right)+\frac{r}{\delta} \frac{\partial \mathcal{T}_{\varepsilon}\left(\widetilde{\mathcal{R}}_{\varepsilon, 3}\right)}{\partial X_{3}} X_{1}\right)+\mathcal{T}_{\varepsilon}^{\prime}\left(\widetilde{e\left(u_{\varepsilon}\right)}\right)_{23},\right. \\
\mathcal{T}_{\varepsilon}^{\prime}\left(\widetilde{e\left(u_{\varepsilon}\right)}\right)_{33}=\frac{1}{\delta} \frac{\partial \mathcal{T}_{\varepsilon}\left(\widetilde{\mathcal{U}}_{\varepsilon, 3}\right)}{\partial X_{3}}+\frac{r}{\delta} \frac{\partial \mathcal{T}_{\varepsilon}\left(\widetilde{\mathcal{R}}_{\varepsilon, 1}\right)}{\partial X_{3}} X_{2}-\frac{r}{\delta} \frac{\partial \mathcal{T}_{\varepsilon}\left(\widetilde{\mathcal{R}}_{\varepsilon, 2}\right)}{\partial X_{3}} X_{1}+\mathcal{T}_{\varepsilon}^{\prime}\left(\widetilde{e\left(u_{\varepsilon}\right)}\right)_{33} .
\end{gathered}
$$

Define the field $\widetilde{u^{\prime}} \in L^{2}\left(\omega \times(0,1), H^{1}\left(D_{1}, \mathbb{R}^{3}\right)\right)$ by

$$
{\widetilde{u^{\prime}}}_{\alpha}=\widetilde{u}_{\alpha}, \quad{\widetilde{u^{\prime}}}_{3}=\widetilde{u}_{3}+X_{1} Z_{1}+X_{2} Z_{2} .
$$

Then

$$
\frac{\partial{\widetilde{u^{\prime}}}_{3}}{\partial X_{1}}=\frac{\partial \widetilde{u}_{3}}{\partial X_{1}}+Z_{1}, \quad \frac{\partial{\widetilde{u^{\prime}}}_{3}}{\partial X_{2}}=\frac{\partial \widetilde{u}_{3}}{\partial X_{2}}+Z_{2}
$$

\section{Appendix: Derivation of the limit problem}

\section{C.1 Equations for the domain $\Omega_{\varepsilon}^{i}$}

\section{Proof. (of Proposition 5.1)}

Step 1. Obtain the limit equations in $\Omega_{\varepsilon}^{i}$.

We will use the following test function:

$$
v_{\varepsilon}(x)=\frac{r}{\delta} \psi(\varepsilon \xi)\left(\begin{array}{c}
\frac{\delta}{r} \varphi_{1}\left(\frac{x_{3}}{\delta}\right)-\frac{x_{2}-\varepsilon \xi_{2}}{r} \varphi_{4}\left(\frac{x_{3}}{\delta}\right) \\
\frac{\delta}{r} \varphi_{2}\left(\frac{x_{3}}{\delta}\right)+\frac{x_{1}-\varepsilon \xi_{1}}{r} \varphi_{4}\left(\frac{x_{3}}{\delta}\right) \\
\varphi_{3}\left(\frac{x_{3}}{\delta}\right)-\frac{x_{1}-\varepsilon \xi_{1}}{r} \frac{d \varphi_{1}}{d X_{3}}\left(\frac{x_{3}}{\delta}\right)-\frac{x_{2}-\varepsilon \xi_{2}}{r} \frac{d \varphi_{2}}{d X_{3}}\left(\frac{x_{3}}{\delta}\right)
\end{array}\right), \quad \xi=\left[\frac{x^{\prime}}{\varepsilon}\right]_{Y},
$$

where $\psi \in C_{c}^{\infty}(\omega), \varphi_{3}$ and $\varphi_{4} \in H_{0}^{1}(0,1), \varphi_{1}$ and $\varphi_{2} \in H_{0}^{2}(0,1)$. Computation of the symmetric strain tensor gives

$$
e\left(v_{\varepsilon}\right)=\frac{r}{\delta^{2}} \psi(\varepsilon \xi)\left(\begin{array}{ccc}
0 & 0 & -\frac{1}{2} \frac{x_{2}-\varepsilon \xi_{2}}{r} \frac{d \varphi_{4}}{d X_{3}} \\
\ldots & 0 & \frac{1}{2} \frac{x_{1}-\varepsilon \xi_{1}}{r} \frac{d \varphi_{4}}{d X_{3}} \\
\ldots & \ldots & \left(\frac{d \varphi_{3}}{d X_{3}}-\frac{x_{1}-\varepsilon \xi_{1}}{r} \frac{d^{2} \varphi_{1}}{d X_{3}^{2}}-\frac{x_{2}-\varepsilon \xi_{2}}{r} \frac{d^{2} \varphi_{2}}{d X_{3}^{2}}\right)
\end{array}\right) \quad \text { in } \varepsilon \xi+B_{1} .
$$

Then

$$
\frac{\delta^{2}}{r} \mathcal{T}_{\varepsilon}^{\prime}\left(\widetilde{e\left(v_{\varepsilon}\right)}\right) \rightarrow \psi\left(x^{\prime}\right)\left(\begin{array}{ccc}
0 & 0 & -\frac{1}{2} X_{2} \frac{d \varphi_{4}}{d X_{3}} \\
\ldots & 0 & \frac{1}{2} X_{1} \frac{d \varphi_{4}}{d X_{3}} \\
\ldots & \ldots & \frac{d \varphi_{3}}{d X_{3}}-X_{1} \frac{d^{2} \varphi_{1}}{d X_{3}^{2}}-X_{2} \frac{d^{2} \varphi_{2}}{d X_{3}^{2}}
\end{array}\right)=V\left(x^{\prime}, X\right) \quad \text { strongly in } L^{2}\left(\omega \times B_{1}\right)
$$

Moreover,

$$
\mathcal{T}_{\varepsilon}^{\prime}\left(\widetilde{v}_{\varepsilon}\right) \rightarrow \psi\left(x^{\prime}\right)\left(\begin{array}{c}
\varphi_{1}\left(X_{3}\right) \\
\varphi_{2}\left(X_{3}\right) \\
0
\end{array}\right) \quad \text { strongly in } L^{2}\left(\omega \times B_{1}\right)
$$

Unfolding the integral over $\Omega_{\varepsilon}^{i}$, we obtain

$$
\begin{aligned}
\int_{\Omega_{\varepsilon}^{i}} \sigma_{\varepsilon}: e\left(v_{\varepsilon}\right) d x & =\sum_{\xi \in \Xi_{\varepsilon}} \int_{\varepsilon \xi+B_{r, \delta}} \sigma_{\varepsilon}: \widetilde{e\left(v_{\varepsilon}\right)} d x \\
& =r^{2} \delta \sum_{\xi \in \Xi_{\varepsilon}} \int_{B_{1}} \mathcal{T}_{\varepsilon}^{\prime}\left(\sigma_{\varepsilon}\right): \mathcal{T}_{\varepsilon}^{\prime}\left(\widetilde{e\left(v_{\varepsilon}\right)}\right) d x^{\prime} d X_{1} d X_{2} d X_{3} \\
& =\frac{r^{2} \delta}{\varepsilon^{2}} \int_{\omega \times B_{1}} \mathcal{T}_{\varepsilon}^{\prime}\left(\sigma_{\varepsilon}\right): \mathcal{T}_{\varepsilon}^{\prime}\left(\widetilde{e\left(v_{\varepsilon}\right)}\right) d x^{\prime} d X_{1} d X_{2} d X_{3}
\end{aligned}
$$


In the same way, integrating the forces we get

$$
\int_{\Omega_{\varepsilon}^{i}} f_{\varepsilon} \cdot v_{\varepsilon} d x=\frac{r^{2} \delta}{\varepsilon^{2}} \int_{\omega \times B_{1}} \mathcal{T}_{\varepsilon}^{\prime}\left(f_{\varepsilon}\right) \cdot \mathcal{T}_{\varepsilon}^{\prime}\left(\widetilde{v}_{\varepsilon}\right) d x^{\prime} d X_{1} d X_{2} d X_{3} .
$$

Taking the limit gives

$$
\frac{\kappa_{0}^{4}}{\kappa_{1}^{3}} \int_{\omega \times B_{1}} \Theta: V d x^{\prime} d X=\sum_{\alpha=1}^{2} \int_{\omega \times B_{1}} F_{\alpha}^{m}\left(x^{\prime}, X\right) \psi\left(x^{\prime}\right) \varphi_{\alpha}(X) d x^{\prime} d X .
$$

We can localize the equation above. This gives

$$
\begin{array}{r}
\frac{\pi \kappa_{0}^{4}}{4 \kappa_{1}^{3}} \mu^{m} \int_{\omega \times(0,1)} \frac{\partial \widetilde{\mathcal{R}}_{3}}{\partial X_{3}} \frac{d \varphi_{4}}{d X_{3}} \psi d x^{\prime} d X_{3}+\frac{\pi \kappa_{0}^{4}}{4 \kappa_{1}^{3}} E^{m} \int_{\omega \times(0,1)}\left(4 \frac{\partial \widetilde{\mathcal{U}}_{3}^{\prime}}{\partial X_{3}} \frac{d \varphi_{3}}{d X_{3}}+\frac{\partial^{2} \widetilde{\mathcal{U}}_{1}}{\partial X_{3}^{2}} \frac{d^{2} \varphi_{1}}{d X_{3}^{2}}+\frac{\partial^{2} \widetilde{\mathcal{U}}_{2}}{\partial X_{3}^{2}} \frac{d^{2} \varphi_{2}}{d X_{3}^{2}}\right) \psi d x^{\prime} d X_{3} \\
=\int_{\omega \times(0,1)}\left(\widetilde{F}_{1}^{m} \varphi_{1}+\widetilde{F}_{2}^{m} \varphi_{2}\right) \psi d x^{\prime} d X_{3} .
\end{array}
$$

The density of the tensor product $\mathcal{C}_{c}^{\infty}(\omega) \otimes H_{0}^{1}(0,1)\left(\operatorname{resp} . \quad \mathcal{C}_{c}^{\infty}(\omega) \otimes H_{0}^{2}(0,1)\right)$ in $L^{2}\left(\omega ; H_{0}^{1}(0,1)\right)($ resp. $\left.L^{2}\left(\omega ; H_{0}^{2}(0,1)\right)\right)$ implies

$$
\begin{aligned}
& \frac{\pi \kappa_{0}^{4}}{4 \kappa_{1}^{3}} \mu^{m} \int_{\omega \times(0,1)} \frac{\partial \widetilde{\mathcal{R}}_{3}}{\partial X_{3}} \frac{\partial \Phi_{4}}{\partial X_{3}} d x^{\prime} d X_{3}+\frac{\pi \kappa_{0}^{4}}{4 \kappa_{1}^{3}} E^{m} \int_{\omega \times(0,1)}\left(4 \frac{\partial \widetilde{\mathcal{U}}_{3}^{\prime}}{\partial X_{3}} \frac{\partial \Phi_{3}}{\partial X_{3}}+\frac{\partial^{2} \widetilde{\mathcal{U}}_{1}}{\partial X_{3}^{2}} \frac{\partial^{2} \Phi_{1}}{\partial X_{3}^{2}}+\frac{\partial^{2} \widetilde{\mathcal{U}}_{2}}{\partial X_{3}^{2}} \frac{\partial^{2} \Phi_{2}}{\partial X_{3}^{2}}\right) d x^{\prime} d X_{3} \\
& =\int_{\omega \times(0,1)}\left(\widetilde{F}_{1}^{m} \Phi_{1}+\widetilde{F}_{2}^{m} \Phi_{2}\right) d x^{\prime} d X_{3} \quad \forall \Phi_{3}, \Phi_{4} \in L^{2}\left(\omega ; H_{0}^{1}(0,1)\right), \quad \forall \Phi_{1}, \Phi_{2} \in L^{2}\left(\omega ; H_{0}^{2}(0,1)\right)
\end{aligned}
$$

Step 2. Obtain $\widetilde{\mathcal{R}}_{3}, \widetilde{\mathcal{U}}_{3}^{\prime}$.

Since $\varphi_{3} \in H_{0}^{1}(0,1)$ is not in the right-hand side of equation (C.2), we obtain

$$
E^{m} \int_{0}^{1} \frac{\partial \widetilde{\mathcal{U}}_{3}^{\prime}}{\partial X_{3}} \frac{d \varphi_{3}}{d X_{3}} d X_{3}=0 \Rightarrow \frac{\partial^{2} \widetilde{\mathcal{U}}_{3}^{\prime}}{\partial X_{3}^{2}}=0 \quad \text { a.e. in } \omega \times(0,1) .
$$

Moreover, we have $\widetilde{\mathcal{U}}_{3}^{\prime}\left(x^{\prime}, 0\right)=0$ for a.e. $x^{\prime} \in \omega$. Therefore, there exists $a \in L^{2}(\omega)$ such that

$$
\tilde{\mathcal{U}}_{3}^{\prime}\left(x^{\prime}, X_{3}\right)=X_{3} a\left(x^{\prime}\right), \quad \text { for a.e. }\left(x^{\prime}, X_{3}\right) \in \omega \times(0,1) .
$$

Similarly, recalling $\varphi_{4} \in H_{0}^{1}(0,1)$ and taking $\varphi_{1}=\varphi_{2}=\varphi_{3}=0$ in (C.2), leads to

$$
\mu^{m} \int_{0}^{1} \frac{\partial \widetilde{\mathcal{R}}_{3}}{\partial X_{3}} \frac{d \varphi_{4}}{d X_{3}} d X_{3}=0 \Rightarrow \frac{\partial^{2} \widetilde{\mathcal{R}}_{3}}{\partial X_{3}^{2}}=0 \quad \text { a.e. in } \omega \times(0,1) .
$$

This together with the boundary conditions (4.4) from Proposition 4.1 gives $\widetilde{\mathcal{R}}_{3}=0$.

The variational problem (5.1) and the boundary conditions (4.9)-(4.10) allow us to determine $\mathcal{U}_{\alpha}(\alpha=1,2)$ in terms of the applied forces $\widetilde{F}_{\alpha}^{m}$ and the traces $u_{\alpha \mid \Sigma}^{ \pm}$.

\section{C.2 Equations for the macroscopic domain}

\section{C.2.1 Determination of $\widetilde{\mathcal{U}}_{3}^{\prime}$}

Proof. (of Lemma 5.1) For the sake of simplicity we extend $\phi$ to a function belonging to $W^{1, \infty}\left(\mathbb{R}^{2}\right)$ which is still denoted by $\phi$. We take

$$
\widetilde{\Xi}_{\varepsilon}=\left\{\xi \in \mathbb{Z}^{2} ;(\varepsilon \xi+\varepsilon Y) \cap \omega \neq \emptyset\right\} .
$$

Observe that $\Xi_{\varepsilon} \subset \widetilde{\Xi}_{\varepsilon}$. Consider the following estimate:

$$
\begin{aligned}
\left\|\phi_{\varepsilon, r}-\phi\right\|_{L^{\infty}(\omega)} & =\left\|\chi\left(\frac{\varepsilon}{r}\left\{\frac{\dot{z}}{\varepsilon}\right\}_{Y}\right)\left(\phi\left(\varepsilon\left[\frac{\dot{z}}{\varepsilon}\right]_{Y}\right)-\phi\right)\right\|_{L^{\infty}(\omega)} \leq \sup _{\xi \in \widetilde{\Xi}_{\varepsilon}}\left\|\chi\left(\frac{\dot{-}}{r}\right)(\phi(\varepsilon \xi)-\phi(\varepsilon \xi+\cdot))\right\|_{L^{\infty}\left(Y_{\varepsilon}\right)} \\
& =\sup _{\xi \in \widetilde{\Xi}_{\varepsilon}}\left\|\chi\left(\frac{\varepsilon}{r} \cdot\right)(\phi(\varepsilon \xi)-\phi(\varepsilon \xi+\varepsilon \cdot))\right\|_{L^{\infty}(Y)} \leq \varepsilon\|\chi\|_{L^{\infty}\left(\mathbb{R}^{2}\right)}\|\nabla \phi\|_{L^{\infty}\left(\mathbb{R}^{2}\right)} .
\end{aligned}
$$


The partial derivative of $\phi_{\varepsilon, r}-\phi$ with respect to $x_{\alpha}$ is

$$
\begin{aligned}
& \frac{\partial\left(\phi_{\varepsilon, r}-\phi\right)}{\partial x_{\alpha}}\left(x^{\prime}\right)=\frac{1}{r} \frac{\partial \chi}{\partial X_{\alpha}}\left(\frac{\varepsilon}{r}\left\{\frac{x^{\prime}}{\varepsilon}\right\}_{Y}\right)\left(\phi\left(\varepsilon\left[\frac{x^{\prime}}{\varepsilon}\right]_{Y}\right)-\phi\left(x^{\prime}\right)\right)-\chi\left(\frac{\varepsilon}{r}\left\{\frac{x^{\prime}}{\varepsilon}\right\}_{Y}\right) \frac{\partial \phi}{\partial x_{\alpha}}\left(x^{\prime}\right), \quad \text { for a.e. } x^{\prime} \in \omega, \\
& \frac{\partial\left(\phi_{\varepsilon, r}-\phi\right)}{\partial x_{\alpha}}\left(\varepsilon \xi+\varepsilon y^{\prime}\right)=\frac{1}{r} \frac{\partial \chi}{\partial X_{\alpha}}\left(\frac{\varepsilon}{r} y^{\prime}\right)\left(\phi(\varepsilon \xi)-\phi\left(\varepsilon \xi+\varepsilon y^{\prime}\right)\right)-\chi\left(\frac{\varepsilon}{r} y^{\prime}\right) \frac{\partial \phi}{\partial x_{\alpha}}\left(\varepsilon \xi+\varepsilon y^{\prime}\right), \quad \xi \in \widetilde{\Xi}_{\varepsilon}, \text { for a.e. } y^{\prime} \in Y .
\end{aligned}
$$

Since $\chi$ has compact support in $\mathbb{R}^{2}$, there exists $R>0$ such that $\operatorname{supp}(\chi) \subset D_{R}$. Thus, the support of the function $y^{\prime} \longmapsto \chi\left(\frac{\varepsilon}{r} y^{\prime}\right)$ is included in the disc $D_{r R / \varepsilon}$. As a consequence we get for a.e. $y^{\prime} \in D_{r R / \varepsilon}$

$$
\left|\phi(\varepsilon \xi)-\phi\left(\varepsilon \xi+\varepsilon y^{\prime}\right)\right| \leq r R\|\nabla \phi\|_{L^{\infty}\left(\mathbb{R}^{2}\right)} .
$$

Using the above estimate, the norms of the derivatives satisfy

$$
\begin{aligned}
\left\|\frac{\partial\left(\phi_{\varepsilon, r}-\phi\right)}{\partial x_{\alpha}}\right\|_{L^{p}(\varepsilon \xi+\varepsilon Y)}^{p} & =\varepsilon^{2}\left\|\frac{\partial \chi}{\partial X_{\alpha}}\left(\frac{\varepsilon}{r} \cdot\right) \frac{\phi(\varepsilon \xi)-\phi(\varepsilon \xi+\varepsilon \cdot)}{r}-\chi\left(\frac{\varepsilon}{r} \cdot\right) \frac{\partial \phi}{\partial x_{\alpha}}(\varepsilon \xi+\varepsilon \cdot)\right\|_{L^{p}(Y)}^{p} \\
& \leq C r^{2}\|\nabla \chi\|_{L^{\infty}\left(\mathbb{R}^{2}\right)}^{p}\|\nabla \phi\|_{L^{\infty}\left(\mathbb{R}^{2}\right)}^{p}
\end{aligned}
$$

The constant $C$ does not depend on $\varepsilon$ and $r$. Combining the above estimates for $\xi \in \widetilde{\Xi}_{\varepsilon}$, that gives

$$
\left\|\nabla\left(\phi_{\varepsilon, r}-\phi\right)\right\|_{L^{p}(\omega)} \leq C\left(\frac{r}{\varepsilon}\right)^{2 / p}\|\nabla \chi\|_{L^{\infty}\left(\mathbb{R}^{2}\right)}\|\nabla \phi\|_{L^{\infty}\left(\mathbb{R}^{2}\right)}
$$

The constant does not depend on $r$ and $\varepsilon$. Hence, estimates (C.5) and (C.6) imply that $\phi_{\varepsilon}$ strongly converges toward $\phi$ in $W^{1, p}(\omega)$.

\section{Proof. (of Lemma 5.2)}

For any $\psi_{3} \in \mathcal{C}^{1}(\bar{\omega} \times[0,1])$ satisfying $\psi_{3}\left(x^{\prime}, 0\right)=0$ for every $x^{\prime} \in \omega$, we consider the following test function:

$$
\begin{aligned}
v_{\varepsilon, \alpha}(x) & =0 \quad \text { for a.e. } x \in \Omega_{\varepsilon}, \quad \alpha=1,2, \\
v_{\varepsilon}(x) & =0 \quad \text { for a.e. } x \in \Omega^{-}, \\
v_{\varepsilon, 3}(x) & =\frac{r}{\delta}\left[\psi_{3}\left(x^{\prime}, 1\right)\left(1-\chi\left(\frac{\varepsilon}{r}\left\{\frac{x^{\prime}}{\varepsilon}\right\}_{Y}\right)\right)+\psi_{3}\left(\varepsilon\left[\frac{x^{\prime}}{\varepsilon}\right]_{Y}, 1\right) \chi\left(\frac{\varepsilon}{r}\left\{\frac{x^{\prime}}{\varepsilon}\right\}_{Y}\right)\right], \quad \text { for a.e. } x \in \Omega_{\varepsilon}^{+}, \\
v_{\varepsilon, 3}(x) & =\frac{r}{\delta} \psi_{3}\left(\varepsilon\left[\frac{x^{\prime}}{\varepsilon}\right]_{Y}, \frac{x_{3}}{\delta}\right), \quad \text { for a.e. } x \in \Omega_{\varepsilon}^{i} .
\end{aligned}
$$

If $\frac{r}{\varepsilon}$ is small enough, $v_{\varepsilon}$ is an admissible test function. The symmetric strain tensor in $\Omega_{\varepsilon}^{i}$ is given by

$$
e\left(v_{\varepsilon}\right)=\frac{r}{\delta^{2}}\left(\begin{array}{ccc}
0 & 0 & 0 \\
\ldots & 0 & 0 \\
\ldots & \ldots & \frac{\partial \psi_{3}}{\partial X_{3}}\left(\varepsilon \xi, \frac{x_{3}}{\delta}\right)
\end{array}\right) \quad \text { a.e. in } \varepsilon \xi+B_{r, \delta}
$$

Then

$$
\frac{\delta^{2}}{r} \mathcal{T}_{\varepsilon}^{\prime}\left(\widetilde{e\left(v_{\varepsilon}\right)}\right) \rightarrow\left(\begin{array}{ccc}
0 & 0 & 0 \\
\ldots & 0 & 0 \\
\ldots & \ldots & \frac{\partial \psi_{3}}{\partial X_{3}}\left(x^{\prime}, X_{3}\right)
\end{array}\right)=V\left(x^{\prime}, X\right) \quad \text { strongly in } L^{2}\left(\omega \times B_{1}\right)
$$

The elements of the symmetric strain tensor in $\Omega_{\varepsilon}^{+}$are written as follows:

$$
\begin{aligned}
& e_{11}\left(v_{\varepsilon}\right)=e_{22}\left(v_{\varepsilon}\right)=e_{12}\left(v_{\varepsilon}\right)=e_{33}\left(v_{\varepsilon}\right)=0, \\
& e_{\alpha 3}\left(v_{\varepsilon}\right)=e_{3 \alpha}\left(v_{\varepsilon}\right)=\frac{1}{2} \frac{r}{\delta} \frac{\partial \psi_{3}}{\partial x_{\alpha}}\left(x^{\prime}, 1\right)(1-\chi(y))+\frac{1}{2 \delta} \frac{\partial \chi}{\partial y_{\alpha}}(y)\left(\psi_{3}\left(x^{\prime}, 1\right)-\psi_{3}\left(\varepsilon\left[\frac{x^{\prime}}{\varepsilon}\right]_{Y}, 1\right)\right),
\end{aligned}
$$

where $y=\frac{\varepsilon}{r}\left\{\frac{x^{\prime}}{\varepsilon}\right\}_{Y}$.

Using Lemma 5.1 and taking into account $\frac{r}{\delta} \rightarrow 0$, the following convergences hold:

$$
\begin{aligned}
& v_{\varepsilon}\left(\cdot+\delta e_{3}\right) \longrightarrow 0 \quad \text { strongly in } H^{1}\left(\Omega^{+} ; \mathbb{R}^{3}\right), \\
& e\left(v_{\varepsilon}\right) \longrightarrow 0 \quad \text { strongly in } L^{2}\left(\Omega^{+} ; \mathbb{R}^{9}\right) .
\end{aligned}
$$


Moreover,

$$
\mathcal{T}_{\varepsilon}^{\prime}\left(v_{\varepsilon}\right) \longrightarrow 0 \quad \text { strongly in } H^{1}\left(\omega \times B_{1} ; \mathbb{R}^{3}\right) .
$$

Using $v_{\varepsilon}$ as a test function in (2.9) and passing to the limit in the unfolded formulation, gives

$$
\int_{\omega \times(0,1)} \frac{\partial \widetilde{\mathcal{U}}_{3}^{\prime}}{\partial X_{3}}\left(x^{\prime}, X_{3}\right) \frac{\partial \psi_{3}}{\partial X_{3}}\left(x^{\prime}, X_{3}\right) d x^{\prime} d X=\int_{\omega \times(0,1)} a\left(x^{\prime}\right) \frac{\partial \psi_{3}}{\partial X_{3}}\left(x^{\prime}, X_{3}\right) d x^{\prime} d X=0 .
$$

Hence $a=0$. Since the test functions are dense in

$$
\mathbb{V}_{s}=\left\{\Psi \in L^{2}\left(\omega ; H^{1}(0,1)\right) \mid \Psi\left(x^{\prime}, 0\right)=0 \text { a.e. in } \omega\right\}
$$

we obtain

$$
\int_{\omega \times(0,1)} \frac{\partial \widetilde{\mathcal{U}}_{3}^{\prime}}{\partial X_{3}}\left(x^{\prime}, X_{3}\right) \frac{\partial \Psi}{\partial X_{3}}\left(x^{\prime}, X_{3}\right) d x^{\prime} d X=0 \quad \forall \Psi \in \mathbb{V}_{s}
$$

\section{C.2.2 Determination of $u_{\alpha}^{ \pm}$and $u_{3}$}

\section{Proof. (of Theorem 5.1)}

For any $v \in \mathbb{V}$ such that $v_{\mid \Omega^{-}} \in W^{1, \infty}\left(\Omega^{-}, \mathbb{R}^{3}\right)$ and $v_{\mid \Omega^{+}} \in W^{1, \infty}\left(\Omega^{+}, \mathbb{R}^{3}\right)$, we first define the displacement $v_{\varepsilon, r}$ in the following way:

$$
v_{\varepsilon, r}(x)=v(x)\left(1-\chi\left(\frac{\varepsilon}{r}\left\{\frac{x^{\prime}}{\varepsilon}\right\}_{Y}\right)\right)+v\left(\varepsilon\left[\frac{x^{\prime}}{\varepsilon}\right]_{Y}, x_{3}\right) \chi\left(\frac{\varepsilon}{r}\left\{\frac{x^{\prime}}{\varepsilon}\right\}_{Y}\right), \quad \text { for a.e. } x \in \Omega^{-} \cup \Omega^{+} .
$$

Then let $h$ denote the following function belonging to $W^{1, \infty}(-L, L)$ :

$$
h\left(x_{3}\right)= \begin{cases}\frac{x_{3}+L}{L}, & x_{3} \in[-L, 0] \\ 1, & x_{3} \geq 0\end{cases}
$$

Now consider the test displacement

$$
\left.\begin{array}{rl}
v_{\varepsilon}^{\prime}(x)= & v(x)\left(1-h\left(x_{3}\right)\right)+v_{\varepsilon, r}(x) h\left(x_{3}\right), \quad \text { for a.e. } x \in \Omega^{-}, \\
v_{\varepsilon}^{\prime}(x)= & v_{\varepsilon, r}\left(x^{\prime}, x_{3}-\delta\right), \quad \text { for a.e. } x \in \Omega_{\varepsilon}^{+}, \\
v_{\varepsilon}^{\prime}(x)= & \psi_{1}\left(\varepsilon\left[\frac{x^{\prime}}{\varepsilon}\right]_{Y}, \frac{x_{3}}{\delta}\right) \\
& \psi_{2}\left(\varepsilon\left[\frac{x^{\prime}}{\varepsilon}\right]_{Y}, \frac{x_{3}}{\delta}\right) \\
v_{3}\left(\varepsilon\left[\frac{x^{\prime}}{\varepsilon}\right]_{Y}, 0\right)-\frac{\varepsilon}{\delta}\left\{\frac{x^{\prime}}{\varepsilon}\right\}_{Y} \cdot \frac{\partial \psi}{\partial X_{3}}\left(\varepsilon\left[\frac{x^{\prime}}{\varepsilon}\right]_{Y}, \frac{x_{3}}{\delta}\right)
\end{array}\right) \quad \text { for a.e. } x \in \Omega_{\varepsilon}^{i},
$$

where $\psi_{\alpha} \in \mathcal{C}^{1}\left(\bar{\omega} ; \mathcal{C}^{3}([0,1])\right), \alpha=1,2$, satisfies

$$
\psi_{\alpha}\left(x^{\prime}, 0\right)=v_{\alpha \mid \Omega^{-}}\left(x^{\prime}, 0\right), \quad \psi_{\alpha}\left(x^{\prime}, 1\right)=v_{\alpha \mid \Omega^{+}}\left(x^{\prime}, 0\right) \quad \text { for every } x^{\prime} \in \omega .
$$

If $\frac{r}{\varepsilon}$ is small enough, $v_{\varepsilon}^{\prime}$ is an admissible test displacement.

Due to Lemma 5.1, the following convergences hold:

$$
\begin{aligned}
& v_{\varepsilon}^{\prime}\left(\cdot+\delta \mathbf{e}_{\mathbf{3}}\right) \longrightarrow v \quad \text { strongly in } H^{1}\left(\Omega^{+} ; \mathbb{R}^{3}\right), \\
& v_{\varepsilon}^{\prime} \longrightarrow v \quad \text { strongly in } H^{1}\left(\Omega^{-} ; \mathbb{R}^{3}\right), \\
& e\left(v_{\varepsilon}^{\prime}\right) \longrightarrow e(v) \quad \text { strongly in } L^{2}\left(\Omega^{+} \cup \Omega^{-} ; \mathbb{R}^{9}\right) .
\end{aligned}
$$

Computing the strain tensor in $\Omega_{\varepsilon}^{i}$ gives

$$
\begin{aligned}
& e_{i j}\left(v_{\varepsilon}^{\prime}\right)=0 \quad(i, j) \neq(3,3) \\
& e_{33}\left(v_{\varepsilon}^{\prime}\right)=-\frac{r}{\delta^{2}}\left(X_{1} \frac{\partial^{2} \psi_{1}}{\partial X_{3}^{2}}\left(\varepsilon\left[\frac{x^{\prime}}{\varepsilon}\right]_{Y}, X_{3}\right)+X_{2} \frac{\partial^{2} \psi_{2}}{\partial X_{3}^{2}}\left(\varepsilon\left[\frac{x^{\prime}}{\varepsilon}\right]_{Y}, X_{3}\right)\right) .
\end{aligned}
$$


Therefore,

$$
\begin{gathered}
\mathcal{T}_{\varepsilon}^{\prime}\left(v_{\varepsilon}^{\prime}\right) \longrightarrow\left(\begin{array}{c}
\psi_{1}\left(x^{\prime}, X_{3}\right) \\
\psi_{2}\left(x^{\prime}, X_{3}\right) \\
v_{3}\left(x^{\prime}, 0\right)
\end{array}\right) \quad \text { strongly in } L^{2}\left(\omega \times B_{1} ; \mathbb{R}^{3}\right), \\
\frac{\delta^{2}}{r} \mathcal{T}_{\varepsilon}^{\prime}\left(e\left(v_{\varepsilon}^{\prime}\right)^{33}\right) \longrightarrow-\left(X_{1} \frac{\partial^{2} \psi_{1}}{\partial X_{3}^{2}}\left(x^{\prime}, X_{3}\right)+X_{2} \frac{\partial^{2} \psi_{2}}{\partial X_{3}^{2}}\left(x^{\prime}, X_{3}\right)\right) \quad \text { strongly in } L^{2}\left(\omega \times B_{1}\right) .
\end{gathered}
$$

Unfolding and taking the limit in $(2.9)$ gives

$$
\begin{aligned}
\int_{\Omega^{ \pm}} \sigma^{ \pm}: e(v) d x-\frac{\kappa_{0}^{4}}{\kappa_{1}^{3}} \int_{\omega \times B_{1}} \Theta:\left(X_{1} \frac{\partial^{2} \psi_{1}}{\partial X_{3}^{2}}+X_{2} \frac{\partial^{2} \psi_{2}}{\partial X_{3}^{2}}\right) d x^{\prime} d X & \\
& =\int_{\Omega^{ \pm}} F v d x+\int_{\omega \times B_{1}}\left(F_{1}^{m} \psi_{1}+F_{2}^{m} \psi_{2}+F_{3} v_{3}\right) d x^{\prime} d X
\end{aligned}
$$

Since the space $W^{1, \infty}\left(\Omega^{+} ; \mathbb{R}^{3}\right)$ is dense in $H^{1}\left(\Omega^{+} ; \mathbb{R}^{3}\right)$, the space of these functions in $W^{1, \infty}\left(\Omega^{-}, \mathbb{R}^{3}\right)$ vanishing on $\Gamma$ is dense in $H^{1}\left(\Omega^{-} ; \mathbb{R}^{3}\right)$ and since the space $\mathcal{C}^{1}\left(\bar{\omega} ; \mathcal{C}^{3}([0,1])\right)$ is dense in $L^{2}\left(\omega ; H^{1}(0,1)\right)$, the equality above holds for every $v$ in $\mathbb{V}$ and every $\psi_{1}, \psi_{2}$ in $L^{2}\left(\omega ; H^{1}(0,1)\right)$ satisfying

$$
\psi_{\alpha}\left(x^{\prime}, 0\right)=v_{\alpha \mid \Omega^{-}}\left(x^{\prime}, 0\right), \quad \psi_{\alpha}\left(x^{\prime}, 1\right)=v_{\alpha \mid \Omega^{+}}\left(x^{\prime}, 0\right) \quad \text { for a.e. } x^{\prime} \in \omega \text {. }
$$

Finally, integrating over $D_{1}$ and making use of (C.3), (C.7) and (5.2) yields the result.

\section{C.2.3 Case (i)}

We introduce the classical unfolding operator.

Definition C.1. For a Lebesgue-measurable function $\varphi$ on $\omega$, the unfolding operator $\mathcal{T}_{\varepsilon}^{\prime \prime}$ is defined as follows:

$$
\mathcal{T}_{\varepsilon}^{\prime \prime}(\varphi)(s, y)=\left\{\begin{array}{lll}
\varphi\left(\varepsilon\left[\frac{s}{\varepsilon}\right]_{Y}+\varepsilon y\right), & \text { for a.e. } & (s, y) \in \widehat{\omega}_{\varepsilon} \times Y, \\
0, & \text { for a.e. } & (s, y) \in \Lambda_{\varepsilon} \times Y .
\end{array}\right.
$$

Recall that (see [8]) the following lemma holds true.

Lemma C.1. Let $\phi$ be in $W^{1, \infty}(\omega)$ and let $\phi_{\varepsilon}$ be defined by

$$
\phi_{\varepsilon}\left(x^{\prime}\right)=\chi\left(\left\{\frac{x^{\prime}}{\varepsilon}\right\}_{Y}\right) \phi\left(\varepsilon\left[\frac{x^{\prime}}{\varepsilon}\right]_{Y}\right)+\left[1-\chi\left(\left\{\frac{x^{\prime}}{\varepsilon}\right\}_{Y}\right)\right] \phi\left(x^{\prime}\right) \quad \text { for a.e. } x^{\prime} \in \omega .
$$

Then we have

$$
\begin{aligned}
& \mathcal{T}_{\varepsilon}^{\prime \prime}\left(\phi_{\varepsilon}\right) \longrightarrow \phi \quad \text { strongly in } L^{2}\left(\omega ; H^{1}(Y)\right), \\
& \mathcal{T}_{\varepsilon}^{\prime \prime}\left(\nabla \phi_{\varepsilon}\right) \longrightarrow \nabla \phi \quad \text { strongly in } L^{2}(\omega \times Y) .
\end{aligned}
$$

Theorem C.1. The variational formulation for the problem (2.9) in the case $(i)$ is given by

$$
\begin{gathered}
\int_{\Omega^{+} \cup \Omega^{-}} \sigma^{ \pm}: e(v) d x+\frac{\pi \kappa_{0}^{4}}{4 \kappa_{1}^{3}} E^{m} \int_{\omega \times(0,1)} \sum_{\alpha=1}^{2} \frac{\partial^{2} \psi_{\alpha}}{\partial X_{3}^{2}} \frac{\partial^{2} \widetilde{\mathcal{U}}_{\alpha}}{\partial X_{3}^{2}} d x^{\prime} d X_{3} \\
+\frac{\pi \kappa_{0}^{4}}{4 \kappa_{1}^{3}} \mu^{m} \int_{\omega \times(0,1)} \frac{\partial \widetilde{\mathcal{R}}_{3}}{\partial X_{3}} \frac{\partial \psi_{4}}{\partial X_{3}} d x^{\prime} d X_{3}+\frac{\pi \kappa_{0}^{4}}{\kappa_{1}^{3}} E^{m} \int_{\omega \times(0,1)} \frac{\partial \widetilde{\mathcal{U}}_{3}^{\prime}}{\partial X_{3}} \frac{\partial \Phi_{3}}{\partial X_{3}} d x^{\prime} d X_{3} \\
=\int_{\Omega^{+} \cup \Omega^{-}} F v d x+\int_{\omega \times(0,1)} \sum_{\alpha=1}^{2} \widetilde{F}_{\alpha}^{m} \psi_{\alpha} d x^{\prime} d X_{3}+\int_{\omega} \bar{F}_{3}^{m} v_{3} d x^{\prime} \\
\forall\left(v, \psi_{1}, \psi_{2}, \psi_{3}, \psi_{4}\right) \in \mathbb{V}_{T}, \quad \forall \Phi_{3} \in L^{2}\left(\omega ; H_{0}^{1}(0,1)\right) .
\end{gathered}
$$

Proof. Step 1. Take the limit in the weak formulation.

In addition to (4.1) and (4.2) we have

$$
\begin{gathered}
\mathcal{T}_{\varepsilon}^{\prime \prime}\left(u_{\varepsilon}\right) \rightarrow u^{-} \quad \text { weakly in } L^{2}\left(\Omega^{-} ; H^{1}(Y)\right), \\
\mathcal{T}_{\varepsilon}^{\prime \prime}\left(\nabla u_{\varepsilon}\right) \rightarrow \nabla u^{-}+\nabla_{y} \widehat{u}^{-} \quad \text { weakly in } L^{2}\left(\Omega^{-} \times Y\right), \\
\mathcal{T}_{\varepsilon}^{\prime \prime}\left(u_{\varepsilon}\right)\left(\cdot+\delta \mathbf{e}_{3}, \cdot \cdot\right) \rightarrow u^{+} \quad \text { weakly in } L^{2}\left(\Omega^{+} ; H^{1}(Y)\right), \\
\mathcal{T}_{\varepsilon}^{\prime \prime}\left(\nabla u_{\varepsilon}\right)\left(\cdot+\delta \mathbf{e}_{3}, \cdot \cdot\right) \rightarrow \nabla u^{+}+\nabla_{y} \widehat{u}^{+} \quad \text { weakly in } L^{2}\left(\Omega^{+} \times Y\right),
\end{gathered}
$$

where $\widehat{u}^{-}$belongs to $L^{2}\left(\Omega^{-} ; H_{p e r}^{1}\left(Y ; \mathbb{R}^{3}\right)\right)$ and $\widehat{u}^{+}$belongs to $L^{2}\left(\Omega^{+} ; H_{p e r}^{1}\left(Y ; \mathbb{R}^{3}\right)\right)$. 
Remark C.1. Here the third variable of $u_{\varepsilon}$ is considered as a parameter, on which the unfolding operator $\mathcal{T}_{\varepsilon}^{\prime \prime}$ does not have any effect.

Step 2. Determination of $\mathcal{U}_{3}^{\prime}$.

To determine the function $a$ introduced in Proposition 5.1, take $\psi_{3} \in \mathcal{C}^{1}(\bar{\omega} \times[0,1])$, satisfying $\psi_{3}\left(x^{\prime}, 0\right)=0$ for every $x^{\prime} \in \omega$, and consider the following test function:

$$
\begin{aligned}
v_{\varepsilon, \alpha}(x) & =0 \quad \text { for a.e. } x \in \Omega_{\varepsilon}, \quad \alpha=1,2, \\
v_{\varepsilon}(x)=0 \quad \text { for a.e. } x \in \Omega^{-}, & \\
v_{\varepsilon, 3}(x) & =\varepsilon^{1 / 3}\left[\psi_{3}\left(x^{\prime}, 1\right)\left(1-\chi\left(\left\{\frac{x^{\prime}}{\varepsilon}\right\}_{Y}\right)\right)+\psi_{3}\left(\varepsilon\left[\frac{x^{\prime}}{\varepsilon}\right]_{Y}, 1\right) \chi\left(\left\{\frac{x^{\prime}}{\varepsilon}\right\}_{Y}\right)\right] \quad \text { for a.e. } x \in \Omega_{\varepsilon}^{+}, \\
v_{\varepsilon, 3}(x) & =\varepsilon^{1 / 3} \psi_{3}\left(\varepsilon\left[\frac{x^{\prime}}{\varepsilon}\right]_{Y}, \frac{x_{3}}{\varepsilon^{2 / 3}}\right) \chi\left(\left\{\frac{x^{\prime}}{\varepsilon}\right\}_{Y}\right) \quad \text { for a.e. } x \in \Omega_{\varepsilon}^{i} .
\end{aligned}
$$

We obtain the following convergences:

$$
\begin{gathered}
v_{\varepsilon}\left(\cdot+\delta \mathbf{e}_{\mathbf{3}}\right) \longrightarrow 0 \quad \text { strongly in } H^{1}\left(\Omega^{+} \cup \Omega^{-} ; \mathbb{R}^{3}\right), \\
e\left(v_{\varepsilon}\right) \longrightarrow 0 \quad \text { strongly in } L^{2}\left(\Omega^{+} \cup \Omega^{-} ; \mathbb{R}^{9}\right), \\
\mathcal{T}_{\varepsilon}^{\prime}\left(v_{\varepsilon}\right) \longrightarrow 0 \quad \text { strongly in } H^{1}\left(\omega \times B_{1} ; \mathbb{R}^{3}\right) .
\end{gathered}
$$

Unfolding and taking the limit as in the Subsection C.2.1 we obtain that $a=0$.

Step 3. For any $v \in \mathbb{V}$ such that $v_{\mid \Omega^{-}} \in W^{1, \infty}\left(\Omega^{-} ; \mathbb{R}^{3}\right)$ and $v_{\mid \Omega^{+}} \in W^{1, \infty}\left(\Omega^{+} ; \mathbb{R}^{3}\right)$, we define the displacement $v_{\varepsilon}$ in the following way:

$$
v_{\varepsilon}(x)=v(x)\left(1-\chi\left(\left\{\frac{x^{\prime}}{\varepsilon}\right\}_{Y}\right)\right)+v\left(\varepsilon\left[\frac{x^{\prime}}{\varepsilon}\right]_{Y}, x_{3}\right) \chi\left(\left\{\frac{x^{\prime}}{\varepsilon}\right\}_{Y}\right), \quad \text { for a.e. } x \in \Omega^{-} \cup \Omega^{+} \text {. }
$$

Consider the following test displacement:

$$
\left.\begin{array}{l}
v_{\varepsilon}^{\prime}(x)=v(x)\left(1-h\left(x_{3}\right)\right)+v_{\varepsilon}(x) h\left(x_{3}\right)+\varepsilon \Psi^{(-)}\left(x^{\prime}, x_{3}\right) \widehat{v}\left(\left\{\frac{x^{\prime}}{\varepsilon}\right\}_{Y}\right), \quad \text { for a.e. } x \in \Omega^{-}, \\
v_{\varepsilon}^{\prime}(x)=v_{\varepsilon}\left(x^{\prime}, x_{3}-\delta\right)+\varepsilon \Psi^{(+)}\left(x^{\prime}, x_{3}-\delta\right) \widehat{v}\left(\left\{\frac{x^{\prime}}{\varepsilon}\right\}_{Y}\right), \quad \text { for a.e. } x \in \Omega_{\varepsilon}^{+}, \\
\psi_{1}\left(\varepsilon\left[\frac{x^{\prime}}{\varepsilon}\right]_{Y}, \frac{x_{3}}{\delta}\right) \\
\psi_{2}\left(\varepsilon\left[\frac{x^{\prime}}{\varepsilon}\right]_{Y}, \frac{x_{3}}{\delta}\right) \\
v_{3}\left(\varepsilon\left[\frac{x^{\prime}}{\varepsilon}\right]_{Y}, 0\right)-\frac{\varepsilon}{\delta}\left\{\frac{x^{\prime}}{\varepsilon}\right\}_{Y} \cdot \frac{\partial \psi}{\partial X_{3}}\left(\varepsilon\left[\frac{x^{\prime}}{\varepsilon}\right]_{Y}, \frac{x_{3}}{\delta}\right)
\end{array}\right) \quad \text { for a.e. } x \in \Omega_{\varepsilon}^{i},
$$

where

- $\widehat{v} \in H_{p e r}^{1}\left(Y ; \mathbb{R}^{3}\right)$,

- $\psi_{\alpha} \in \mathcal{C}^{1}\left(\bar{\omega} ; \mathcal{C}^{3}([0,1])\right), \alpha=1,2$, satisfies

$$
\psi_{\alpha}\left(x^{\prime}, 0\right)=v_{\alpha \mid \Omega^{-}}\left(x^{\prime}, 0\right), \quad \psi_{\alpha}\left(x^{\prime}, 1\right)=v_{\alpha \mid \Omega^{+}}\left(x^{\prime}, 0\right) \quad \text { for every } x^{\prime} \in \omega,
$$

- $\Psi^{(-)} \in W^{1, \infty}\left(\Omega^{-}\right), \Psi^{(+)} \in W^{1, \infty}\left(\Omega^{+}\right)$satisfies

$$
\Psi^{( \pm)}\left(x^{\prime}, 0\right)=0, \quad \text { a.e. in } \omega, \quad \Psi^{(-)}=0 \quad \text { on } \Gamma
$$

- $h\left(x_{3}\right)$ is defined as in (C.9).

Using (5.2), we obtain the following convergences:

$$
\begin{aligned}
& \mathcal{T}_{\varepsilon}^{\prime \prime}\left(v_{\varepsilon}^{\prime}(\cdot, \cdot \cdot)\right) \longrightarrow v \quad \text { strongly in } L^{2}\left(\Omega^{-} ; H^{1}(Y)\right), \\
& \mathcal{T}_{\varepsilon}^{\prime \prime}\left(\nabla v_{\varepsilon}^{\prime}(\cdot, \cdot \cdot)\right) \longrightarrow \nabla v+\Psi^{(-)} \nabla_{y} \widehat{v} \quad \text { strongly in } L^{2}\left(\Omega^{-} \times Y\right), \\
& \mathcal{T}_{\varepsilon}^{\prime \prime}\left(v_{\varepsilon}^{\prime}\left(\cdot+\delta \mathbf{e}_{3}, \cdot \cdot\right)\right) \longrightarrow v \quad \text { strongly in } L^{2}\left(\Omega^{+} ; H^{1}(Y)\right), \\
& \mathcal{T}_{\varepsilon}^{\prime \prime}\left(\nabla v_{\varepsilon}^{\prime}\left(\cdot+\delta \mathbf{e}_{3}, \cdot \cdot\right)\right) \longrightarrow \nabla v+\Psi^{(+)} \nabla_{y} \widehat{v} \quad \text { strongly in } L^{2}\left(\Omega^{+} \times Y\right) .
\end{aligned}
$$


Moreover,

$$
\begin{gathered}
\mathcal{T}_{\varepsilon}\left(\mathcal{T}_{\varepsilon}^{\prime \prime}\left(v_{\varepsilon}^{\prime}\right)\right) \longrightarrow\left(\begin{array}{c}
\psi_{1}\left(x^{\prime}, X_{3}\right) \\
\psi_{2}\left(x^{\prime}, X_{3}\right) \\
v_{3}\left(x^{\prime}, 0\right)
\end{array}\right) \quad \text { strongly in } L^{2}\left(\omega ; H^{1}\left(Y \times B_{1}\right)\right), \\
\frac{\delta^{2}}{r} \mathcal{T}_{\varepsilon}\left(\mathcal{T}_{\varepsilon}^{\prime \prime}\left(e_{33}\left(v_{\varepsilon}^{\prime}\right)\right)\right) \longrightarrow-X_{1} \frac{\partial^{2} \psi_{1}}{\partial X_{3}^{2}}\left(x^{\prime}, X_{3}\right)-X_{2} \frac{\partial^{2} \psi_{2}}{\partial X_{3}^{2}}\left(x^{\prime}, X_{3}\right) \quad \text { strongly in } L^{2}\left(\omega \times Y \times B_{1}\right) .
\end{gathered}
$$

Unfolding and taking the limit, we obtain

$$
\begin{aligned}
\int_{\Omega^{ \pm} \times Y}\left(\sigma^{ \pm}+\widehat{\sigma}^{ \pm}\right):\left(e(v)+\Psi^{( \pm)} e_{y}(\widehat{v})\right) d x d y & -\frac{\kappa_{0}^{4}}{\kappa_{1}^{3}} \int_{\omega \times B_{1}} \Theta:\left(X_{1} \frac{\partial^{2} \psi_{1}}{\partial X_{3}^{2}}+X_{2} \frac{\partial^{2} \psi_{2}}{\partial X_{3}^{2}}\right) d x^{\prime} d X= \\
& =\int_{\Omega^{ \pm}} F v d x+\int_{\omega \times B_{1}}\left(F_{1}^{m} \psi_{1}+F_{2}^{m} \psi_{2}+F_{3} v_{3}\right) d x^{\prime} d X
\end{aligned}
$$

Since $\sigma^{ \pm}$and $e(v)$ do not depend on $y$ and due to the periodicity of the fields $\widehat{v}$ and $\widehat{u}^{ \pm}$, the equality above reads

$$
\begin{aligned}
\int_{\Omega^{ \pm}} \sigma^{ \pm}: e(v) d x+\int_{\Omega^{ \pm} \times Y} \widehat{\sigma}^{ \pm}: \Psi^{( \pm)} e_{y}(\widehat{v}) d x d y-\frac{\kappa_{0}^{4}}{\kappa_{1}^{3}} \int_{\omega \times B_{1}} \Theta:\left(X_{1} \frac{\partial^{2} \psi_{1}}{\partial X_{3}^{2}}+X_{2} \frac{\partial^{2} \psi_{2}}{\partial X_{3}^{2}}\right) d x^{\prime} d X= \\
=\int_{\Omega^{ \pm}} F v d x+\int_{\omega \times B_{1}}\left(F_{1}^{m} \psi_{1}+F_{2}^{m} \psi_{2}+F_{3} v_{3}\right) d x^{\prime} d X
\end{aligned}
$$

Step 3. To determine $\widehat{\sigma}$ we first take $v=0$. We then obtain

$$
\int_{\Omega^{ \pm} \times Y} \widehat{\sigma}^{ \pm}: \Psi^{( \pm)} e_{y}(\widehat{v}) d x d y-\frac{\kappa_{0}^{4}}{\kappa_{1}^{3}} \int_{\omega \times B_{1}} \Theta:\left(X_{1} \frac{\partial^{2} \psi_{1}}{\partial X_{3}^{2}}+X_{2} \frac{\partial^{2} \psi_{2}}{\partial X_{3}^{2}}\right) d x^{\prime} d X=\int_{\omega \times B_{1}}\left(F_{1}^{m} \psi_{1}+F_{2}^{m} \psi_{2}\right) d x^{\prime} d X
$$

Since the right-hand side does not contain $\widehat{v}$,

$$
\int_{\Omega^{ \pm} \times Y} \widehat{\sigma}^{ \pm}: \Psi^{( \pm)} e_{y}(\widehat{v}) d x d y=0
$$

which corresponds to the strong formulation

$$
\begin{cases}\sum_{j=1}^{3} \frac{\partial \widehat{\sigma}_{i j}^{ \pm}}{\partial y_{j}}=0, & \text { in } \Omega^{ \pm} \times Y, \\ \sum_{j=1}^{3} \widehat{\sigma}_{i j}^{ \pm}=0, & \text { on } \partial\left(\Omega^{ \pm} \times Y\right),\end{cases}
$$

for $i=1,2,3$. Therefore, $\widehat{\sigma}^{ \pm}=0$, and (C.16) is rewritten as

$$
\begin{aligned}
\int_{\Omega^{ \pm}} \sigma^{ \pm}: e(v) d x-\frac{\kappa_{0}^{4}}{\kappa_{1}^{3}} \int_{\omega \times B_{1}} \Theta:\left(X_{1} \frac{\partial^{2} \psi_{1}}{\partial X_{3}^{2}}\right. & \left.=X_{2} \frac{\partial^{2} \psi_{2}}{\partial X_{3}^{2}}\right) d x^{\prime} d X= \\
& =\int_{\Omega^{ \pm}} F v d x+\int_{\omega \times B_{1}}\left(F_{1}^{m} \psi_{1}+F_{2}^{m} \psi_{2}+F_{3} v_{3}\right) d x^{\prime} d X
\end{aligned}
$$

Since the space $W^{1, \infty}\left(\Omega^{+} ; \mathbb{R}^{3}\right)$ is dense in $H^{1}\left(\Omega^{+} ; \mathbb{R}^{3}\right)$, since the space of functions in $W^{1, \infty}\left(\Omega^{-}, \mathbb{R}^{3}\right)$ vanishing on $\Gamma$ is dense in $H^{1}\left(\Omega^{-} ; \mathbb{R}^{3}\right)$ and since the space $\mathcal{C}^{1}\left(\bar{\omega} ; \mathcal{C}^{3}([0,1])\right)$ is dense in $L^{2}\left(\omega ; H^{1}(0,1)\right)$, the equality above holds for every $v$ in $\mathbb{V}$ and every $\psi_{1}, \psi_{2}$ in $L^{2}\left(\omega ; H^{1}(0,1)\right)$ satisfying

$$
\psi_{\alpha}\left(x^{\prime}, 0\right)=v_{\alpha \mid \Omega^{-}}\left(x^{\prime}, 0\right), \quad \psi_{\alpha}\left(x^{\prime}, 1\right)=v_{\alpha \mid \Omega^{+}}\left(x^{\prime}, 0\right) \quad \text { for a.e. } x^{\prime} \in \omega \text {. }
$$

Finally, integrating over $D_{1}$ and using (5.2), we obtain the result.

\section{C.3 Convergences}

Theorem C.2. Under the assumptions (3.22) on the applied forces, we first have (convergence of the stress energy)

$$
\lim _{\varepsilon \rightarrow 0} \mathcal{E}\left(u_{\varepsilon}\right)=\int_{\Omega^{+} \cup \Omega^{-}} \sigma^{ \pm}: e(u) d x+\frac{\pi \kappa_{0}^{4}}{4 \kappa_{1}^{3}} E^{m} \int_{\omega \times(0,1)} \sum_{\alpha=1}^{2}\left|\frac{\partial^{2} \widetilde{\mathcal{U}}_{\alpha}}{\partial X_{3}^{2}}\right|^{2} d x^{\prime} d X_{3} .
$$

The sequence $\left(u_{\varepsilon}, \sigma_{\varepsilon}\right)$ shows the following convergence behavior: 
- $u_{\varepsilon} \rightarrow u^{-}$strongly in $H^{1}\left(\Omega^{-}\right)$,

$u_{\varepsilon}\left(\cdot+\delta \mathbf{e}_{3}\right) \rightarrow u^{+}$strongly in $H^{1}\left(\Omega^{+}\right)$,

- $\sigma_{\varepsilon} \rightarrow \sigma^{-}$strongly in $L^{2}\left(\Omega^{-}\right)$,

$\sigma_{\varepsilon}\left(\cdot+\delta \mathbf{e}_{3}\right) \rightarrow \sigma^{+}$strongly in $L^{2}\left(\Omega^{+}\right)$,

- $\frac{\delta^{2}}{r} \mathcal{T}_{\varepsilon}^{\prime}\left(\sigma_{\varepsilon}\right) \rightarrow \Theta$ strongly in $L^{2}\left(\omega \times B_{1}\right)$, where

$$
\Theta_{i j}=\left\{\begin{array}{lr}
-E^{m}\left(X_{1} \frac{\partial^{2} \widetilde{\mathcal{U}}_{1}}{\partial X_{3}^{2}}+X_{2} \frac{\partial^{2} \widetilde{\mathcal{U}}_{2}}{\partial X_{3}^{2}}\right), & (i, j)=(3,3), \\
0, & \text { otherwise }
\end{array}\right.
$$

- $\delta \mathcal{T}_{\varepsilon}\left(\widetilde{\mathcal{R}}_{\varepsilon, \alpha}\right) \rightarrow \widetilde{\mathcal{R}}_{\alpha}, \quad \alpha=1,2$ strongly in $L^{2}\left(\omega ; H^{1}(0,1)\right)$,

- $\mathcal{T}_{\varepsilon}\left(\widetilde{\mathcal{U}}_{\varepsilon, \alpha}\right) \rightarrow \widetilde{\mathcal{U}}_{\alpha}, \quad \alpha=1,2$ strongly in $L^{2}\left(\omega ; H^{1}(0,1)\right)$,

$\mathcal{T}_{\varepsilon}\left(\widetilde{\mathcal{U}}_{\varepsilon, 3}\right) \rightarrow u_{3}^{ \pm}(\cdot, 0)$ strongly in $L^{2}\left(\omega ; H^{1}(0,1)\right)$,

$\frac{\delta}{r} \mathcal{T}_{\varepsilon}\left(\widetilde{\mathcal{U}}_{\varepsilon, 3}-\widetilde{\mathcal{U}}_{\varepsilon, 3}(\cdot, \cdot, 0)\right) \rightarrow 0$ strongly in $L^{2}\left(\omega ; H^{1}(0,1)\right)$.

Proof. Step 1. We prove (C.18).

We first recall a classical identity: if $T$ is a symmetric $3 \times 3$ matrix we have

$$
\begin{aligned}
\lambda^{m} \operatorname{Tr}(T) \operatorname{Tr}(T)+\sum_{i, j=1}^{3} 2 \mu^{m} T_{i j} T_{i j} & =E^{m} T_{33}^{2}+\frac{E^{m}}{\left(1+\nu^{m}\right)\left(1-2 \nu^{m}\right)}\left(T_{11}+T_{22}+2 \nu^{m} T_{33}\right)^{2} \\
& +\frac{E^{m}}{2\left(1+\nu^{m}\right)}\left[\left(T_{11}-T_{22}\right)^{2}+4\left(T_{12}^{2}+T_{13}^{2}+T_{23}^{2}\right)\right] .
\end{aligned}
$$

Now, we consider the total elastic energy of the displacement $u_{\varepsilon}$, given by $(2.10)$ :

$$
\mathcal{E}\left(u_{\varepsilon}\right)=\int_{\Omega_{\varepsilon}} \sigma_{\varepsilon}: e\left(u_{\varepsilon}\right) d x=\int_{\Omega_{\varepsilon}} f_{\varepsilon} \cdot u_{\varepsilon} d x
$$

The left-hand side of (C.20) is

$$
\begin{aligned}
\mathcal{E}\left(u_{\varepsilon}\right) & =\int_{\Omega_{\varepsilon}} \sigma_{\varepsilon}: e\left(u_{\varepsilon}\right) d x \\
& =\int_{\Omega^{-}} \sigma_{\varepsilon}: e\left(u_{\varepsilon}\right) d x+\int_{\Omega_{\varepsilon}^{i}} \sigma_{\varepsilon}: e\left(u_{\varepsilon}\right) d x+\int_{\Omega_{\varepsilon}^{+}} \sigma_{\varepsilon}: e\left(u_{\varepsilon}\right) d x \\
& =\int_{\Omega^{-}} \sigma_{\varepsilon}: e\left(u_{\varepsilon}\right) d x+\frac{r^{2} \delta}{\varepsilon^{2}} \int_{\omega \times B_{1}} \mathcal{T}_{\varepsilon}^{\prime}\left(\sigma_{\varepsilon}\right): \mathcal{T}_{\varepsilon}^{\prime}\left(e\left(u_{\varepsilon}\right)\right) d x^{\prime} d X+\int_{\Omega_{\varepsilon}^{+}} \sigma_{\varepsilon}: e\left(u_{\varepsilon}\right) d x .
\end{aligned}
$$

The second term of the right-hand side in the equation above is transformed using identity (C.19):

$$
\begin{aligned}
& \int_{\omega \times B_{1}} \mathcal{T}_{\varepsilon}^{\prime}\left(\sigma_{\varepsilon}\right): \mathcal{T}_{\varepsilon}^{\prime}\left(e\left(u_{\varepsilon}\right)\right) d x^{\prime} d X \\
= & \int_{\omega \times B_{1}}\left(E^{m} e_{33}^{2}\left(u_{\varepsilon}\right)+\frac{E^{m}}{\left(1+\nu^{m}\right)\left(1-2 \nu^{m}\right)}\left(e_{11}\left(u_{\varepsilon}\right)+e_{22}\left(u_{\varepsilon}\right)+2 \nu^{m} e_{33}\left(u_{\varepsilon}\right)\right)^{2}\right. \\
+ & \left.\frac{E^{m}}{2\left(1+\nu^{m}\right)}\left[\left(e_{11}\left(u_{\varepsilon}\right)-e_{22}\left(u_{\varepsilon}\right)\right)^{2}+4\left(\left(e_{12}\left(u_{\varepsilon}\right)\right)^{2}+\left(e_{13}\left(u_{\varepsilon}\right)\right)^{2}+\left(e_{23}\left(u_{\varepsilon}\right)\right)^{2}\right)\right]\right) d x^{\prime} d X .
\end{aligned}
$$

Then by standard weak lower semi-continuity, Lemma 4.1 and (4.16) give (we recall that $\widetilde{\mathcal{R}}_{3}=0$ )

$$
\int_{\Omega^{+} \cup \Omega^{-}} \sigma^{ \pm}: e(u) d x+\frac{\kappa_{0}^{4}}{\kappa_{1}^{3}} \int_{\omega \times B_{1}} E^{m} e_{33}^{2}(u) d x^{\prime} d X \leq \liminf _{\varepsilon \rightarrow 0} \mathcal{E}\left(u_{\varepsilon}\right) .
$$

Besides, the convergences in Proposition 4.1 lead to

$$
\begin{aligned}
\limsup _{\varepsilon \rightarrow 0} \mathcal{E}\left(u_{\varepsilon}\right) & =\limsup _{\varepsilon \rightarrow 0} \int_{\Omega_{\varepsilon}} f_{\varepsilon} \cdot u_{\varepsilon} d x=\lim _{\varepsilon \rightarrow 0} \int_{\Omega_{\varepsilon}} f_{\varepsilon} \cdot u_{\varepsilon} d x \\
& =\int_{\Omega^{+} \cup \Omega^{-}} F \cdot u d x+\int_{\omega \times(0,1)} \sum_{\alpha=1}^{2} \widetilde{F}_{\alpha}^{m} \widetilde{\mathcal{U}}_{\alpha} d x^{\prime} d X_{3}+\int_{\omega} \bar{F}_{3}^{m} u_{3} d x^{\prime} .
\end{aligned}
$$


Hence

$$
\limsup _{\varepsilon \rightarrow 0} \mathcal{E}\left(u_{\varepsilon}\right) \leq \liminf _{\varepsilon \rightarrow 0} \mathcal{E}\left(u_{\varepsilon}\right)
$$

Therefore (we recall that $\widetilde{\mathcal{U}}_{3}^{\prime}=0$ ),

$$
\lim _{\varepsilon \rightarrow 0} \mathcal{E}\left(u_{\varepsilon}\right)=\int_{\Omega^{+} \cup \Omega^{-}} \sigma^{ \pm}: e(u) d x+\frac{\pi \kappa_{0}^{4}}{4 \kappa_{1}^{3}} E^{m} \int_{\omega \times(0,1)} \sum_{\alpha=1}^{2}\left|\frac{\partial^{2} \widetilde{\mathcal{U}}_{\alpha}}{\partial X_{3}^{2}}\right|^{2} d x^{\prime} d X_{3}
$$

Step 2. As an immediate consequence of the convergence (C.23) above, we have

$$
\begin{aligned}
& \sigma_{\varepsilon} \rightarrow \sigma^{-} \quad \text { strongly in } L^{2}\left(\Omega^{-}\right), \\
& \sigma_{\varepsilon}\left(\cdot+\delta \mathbf{e}_{3}\right) \rightarrow \sigma^{+} \quad \text { strongly in } L^{2}\left(\Omega^{+}\right), \\
& \frac{\delta^{2}}{r} \mathcal{T}_{\varepsilon}^{\prime}\left(\sigma_{\varepsilon}\right) \rightarrow \Theta \quad \text { strongly in } L^{2}\left(\omega \times B_{1}\right) .
\end{aligned}
$$

Hence

$$
\begin{aligned}
& e\left(u_{\varepsilon}\right) \rightarrow e\left(u^{-}\right) \quad \text { strongly in } L^{2}\left(\Omega^{-}\right), \\
& e\left(u_{\varepsilon}\left(\cdot+\delta \mathbf{e}_{3}\right)\right) \rightarrow e\left(u^{+}\right) \quad \text { strongly in } L^{2}\left(\Omega^{+}\right), \\
& \frac{\delta^{2}}{r} \mathcal{T}_{\varepsilon}^{\prime}\left(\widetilde{e\left(u_{\varepsilon}\right)}\right) \rightarrow X \quad \text { strongly in } L^{2}\left(\omega \times B_{1}\right) .
\end{aligned}
$$

From (C.25) ${ }_{1}$ and Korn's inequality (2.11) in $\Omega^{-}$, we deduce that

$$
u_{\varepsilon} \rightarrow u^{-} \text {strongly in } H^{1}\left(\Omega^{-}\right) \text {. }
$$

The strong convergence above and the estimates (3.9)-(3.10) yield

$$
u_{\varepsilon}(\cdot, 0) 1_{\widehat{\omega}_{\varepsilon}} \rightarrow u_{\mid \Sigma}^{-}=\widetilde{\mathcal{U}}(\cdot, 0) \text { strongly in } L^{2}(\Sigma)
$$

From $\left(\right.$ C.25) 3 and $(4.15)_{4}$ we derive that

$$
\frac{\delta}{r} \frac{\partial \mathcal{T}_{\varepsilon}\left(\widetilde{\mathcal{U}}_{\varepsilon, 3}\right)}{\partial X_{3}}+\delta \frac{\partial \mathcal{T}_{\varepsilon}\left(\widetilde{\mathcal{R}}_{\varepsilon, 1}\right)}{\partial X_{3}} X_{2}-\delta \frac{\partial \mathcal{T}_{\varepsilon}\left(\widetilde{\mathcal{R}}_{\varepsilon, 2}\right)}{\partial X_{3}} X_{1}+\frac{\delta^{2}}{r} \mathcal{T}_{\varepsilon}^{\prime}\left(\left(\widetilde{e\left(u_{\varepsilon}\right)}\right)_{33} \rightarrow \frac{\partial \widetilde{\mathcal{R}}_{1}}{\partial X_{3}} X_{2}-\frac{\partial \widetilde{\mathcal{R}}_{2}}{\partial X_{3}} X_{1}\right.
$$

Hence, the equalities $(3.4)_{1}-(3.4)_{3}$ with the estimates $(3.8)_{1}-(3.11)$ and the convergence in (C.26) lead to

$$
\begin{gathered}
\delta \mathcal{T}_{\varepsilon}\left(\widetilde{\mathcal{R}}_{\varepsilon, \alpha}\right) \rightarrow \widetilde{\mathcal{R}}_{\alpha}, \quad \alpha=1,2 \quad \text { strongly in } L^{2}\left(\omega ; H^{1}(0,1)\right), \\
\frac{\delta}{r} \mathcal{T}_{\varepsilon}\left(\widetilde{\mathcal{U}}_{\varepsilon, 3}-\widetilde{\mathcal{U}}_{\varepsilon, 3}(\cdot, \cdot, 0)\right) \rightarrow 0 \quad \text { strongly in } L^{2}\left(\omega ; H^{1}(0,1)\right), \\
\mathcal{T}_{\varepsilon}\left(\widetilde{\mathcal{U}}_{\varepsilon, 3}\right) \rightarrow u_{3 \mid \Sigma}^{-}=u_{3 \mid \Sigma}^{+} \quad \text { strongly in } L^{2}\left(\omega, H^{1}(0,1)\right) .
\end{gathered}
$$

The fourth estimate in Lemma B.2, the convergences in (C.26)-(C.27) and the equalities (4.11) imply that

$$
\mathcal{T}_{\varepsilon}\left(\widetilde{\mathcal{U}}_{\varepsilon, \alpha}\right) \rightarrow \widetilde{\mathcal{U}}_{\alpha}, \quad \alpha=1,2 \quad \text { strongly in } L^{2}\left(\omega ; H^{1}(0,1)\right)
$$

From the convergences in (C.29)-(C.30) and the estimates (3.11)-(3.12) we obtain

$$
u_{\varepsilon}(\cdot, \delta) 1_{\widehat{\omega}_{\varepsilon}} \rightarrow u_{\mid \Sigma}^{+}=\widetilde{\mathcal{U}}(\cdot, 1) \text { strongly in } L^{2}(\Sigma) .
$$

Finally, due to the strong convergence above together with $\left(\right.$ C.25) 2 and $(\mathrm{A} .17)_{2}$, we get $u_{\varepsilon}\left(\cdot+\delta \mathbf{e}_{\mathbf{3}}\right) \rightarrow u^{+}$ strongly in $H^{1}\left(\Omega^{+}\right)$.

\section{References}

[1] S. Antman, Theory of rods, Handbuch der Physik, Band VIa/2, pp. 641-703. Springer, Berlin, 1972

[2] D. Blanchard, A. Gaudiello, G. Griso Junction of a periodic family of elastic rods with a 3d plate. Part I, J. Math. Pures Appl., Vol. 88-1, pp. 1-33, 2007 
[3] D. Blanchard, A. Gaudiello, G. Griso Junction of a periodic family of elastic rods with a 3d plate. Part II, J. Math. Pures Appl., Vol. 88-2, pp. 149-190, 2007

[4] D. Blanchard, G. Griso Junction between a plate and a rod of comrarable thickness in non-linear elasticity, J. Elast. (2013) Volume 112, Num. 2, pp. 79-109, 2013

[5] P. Ciarlet, Mathematical elasticity, Vol.I, North-Holand, Amsterdam, 1988

[6] P. Ciarlet, Mathematical elasticity, Vol.II: Theory of plates. North-Holand, Amsterdam, 1997

[7] D. Cioranescu, A. Damlamian, G. Griso, D. Onofrei The periodic unfolding method for perforated domains and Neumann sieve models, J. Math. Pures Appl., Vol. 89, 2008

[8] D. Cioranescu, A. Damlamian, G. Griso The periodic unfolding method in homogenization, SIAM J. of Math. Anal. Vol. 40, 4, pp. 1585-1620, 2008

[9] G. Geymonat, F. Krasucki, S. Lenci Mathematical analysis of a bonded joint with a soft thin adhesive, Math. Mech. Solids, Vol. 4, (1999), pp. 201-225

[10] G. Griso Decompositions of displacements of thin structures, J. Math. Pures Appl., Vol. 89, pp. 199-223, 2008

[11] G. Griso Asymptotic behavior of curved rods by the unfolding method, Math. Meth. Appl. Sci., Vol. 27, pp. 2081-2110, 2004

[12] M. Neuss-Radu and W. Jäger Effective transmission conditions for reaction-diffusion processes in domains separated by an interface, SIAM J. Math. Anal., Vol. 39, No. 3, pp. 687-720, 2007

[13] O. A. Oleinik, A. S. Shamaev, G. A. Yosifan Mathematical Problems in Elasticity and Homogenization, North Holland, 1992

[14] L. Trabucho, J. M. Viano Mathematical Modelling of Rods, Hand-book of Numerical Analysis, Vol. 4, North-Holland, Amsterdam, 1996 